\title{
Impacts of Metabolism and Organic Acids on Cell Wall Composition and Pseudomonas aeruginosa Susceptibility to Membrane Active Antimicrobials
}

Giorgia Manzo, Federico Gianfanti, Charlotte K. Hind, Leanne Allison, Maria Clarke, Julia Hohenbichler, Ilene Limantoro, Bethany Martin, Phoebe Do Carmo Silva, Philip M. Ferguson, Alice C. Hodgson-Casson, Roland A. Fleck, J. Mark Sutton, David A. Phoenix, and A. James Mason*

Cite This: https://doi.org/10.1021/acsinfecdis.1c00002

ABSTRACT: Reliable antimicrobial susceptibility testing is essential in informing both clinical antibiotic therapy decisions and the development of new antibiotics. Mammalian cell culture media have been proposed as an alternative to bacteriological media, potentially representing some critical aspects of the infection environment more accurately. Here, we use a combination of NMR metabolomics and electron microscopy to investigate the response of Escherichia coli and Pseudomonas aeruginosa to growth in differing rich media to determine whether and how this determines metabolic strategies, the composition of the cell wall, and consequently susceptibility to membrane active antimicrobials including colistin and tobramycin. The NMR
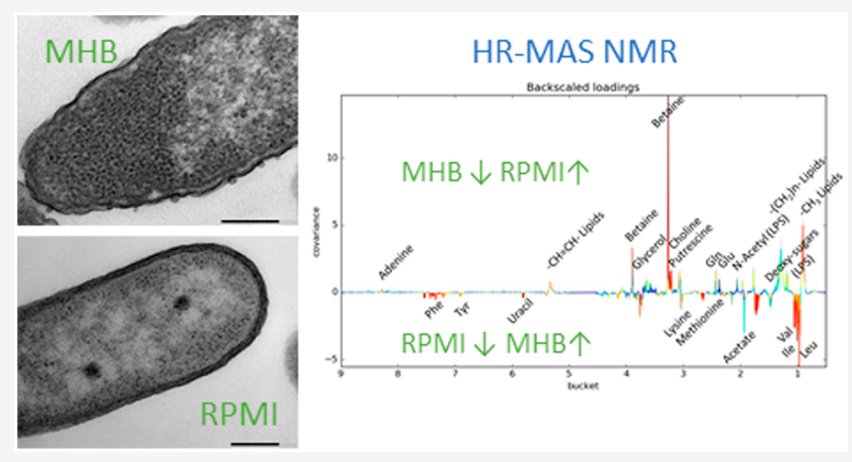
metabolomic approach is first validated by characterizing the expected E. coli acid stress response to fermentation and the accompanying changes in the cell wall composition, when cultured in glucose rich mammalian cell culture media. Glucose is not a major carbon source for $P$. aeruginosa but is associated with a response to osmotic stress and a modest increase in colistin tolerance. Growth of P. aeruginosa in a range of bacteriological media is supported by consumption of formate, an important electron donor in anaerobic respiration. In mammalian cell culture media, however, the overall metabolic strategy of $P$. aeruginosa is instead dependent on consumption of glutamine and lactate. Formate doping of mammalian cell culture media does not alter the overall metabolic strategy but is associated with polyamine catabolism, remodelling of both inner and outer membranes, and a modest sensitization of $P$. aeruginosa PAO1 to colistin. Further, in a panel of $P$. aeruginosa isolates an increase between 2- and 3-fold in sensitivity to tobramycin is achieved through doping with other organic acids, notably propionate which also similarly enhances the activity of colistin. Organic acids are therefore capable of nonspecifically influencing the potency of membrane active antimicrobials.

KEYWORDS: antimicrobial susceptibility testing, NMR metabolomics, colistin

$\mathrm{T}$ he effective development of new antibiotics to combat the rise in infections due to antimicrobial resistant bacteria is dependent on antimicrobial susceptibility screening being a reliable predictor of therapeutic outcomes. Recently the use of the universal media standard Mueller-Hinton Broth (MHB) for in vitro efficacy testing, as recommended by the Clinical and Laboratory Standards Institute, ${ }^{1}$ has been challenged since this does not replicate the nutritional environment provided by the host and hence does not predict patient outcomes. The use of MHB can produce both false-positive and false-negative results. For example, Salmonella has been shown to be sensitive to polymyxin in MHB but it induces polymyxin resistance during infection. ${ }^{2}$ In contrast, azithromycin, which lacks activity against Gram-negative bacteria in $\mathrm{MHB}$, was shown to be effective against multidrug resistant (MDR) strains of Pseudomonas aeruginosa, Klebsiella pneumoniae, and Acinetobacter baumannii in eukaryotic cell culture media RPMI-1640 and in murine infection models. ${ }^{3}$ Consequently, antibacterial testing has been conducted in a variety of cell culture media to identify readily available media that generate results that are more predictive of in vivo therapeutic outcomes. ${ }^{4}$

A complementary approach has been to work to define the key elements of the host nutritional environment from the bacterial

Received: January 3, 2021 
Table 1. Effect of Media on Susceptibility to Membrane Active Antimicrobials ${ }^{a}$

\begin{tabular}{|c|c|c|c|c|c|c|c|c|}
\hline & \multicolumn{4}{|c|}{ E. coli NCTC 9001} & \multicolumn{4}{|c|}{ P. aeruginosa $\mathrm{PAO} 1$} \\
\hline & LL-37 & Pleurocidin & Magainin 2 & Colistin & LL-37 & Pleurocidin & Magainin 2 & Colistin \\
\hline MHB & $1.48 \pm 0.19$ & $0.90 \pm 0.07$ & $1.76 \pm 0.05$ & $0.12 \pm 0.01$ & $7.21 \pm 0.59$ & $6.02 \pm 0.11$ & $30.30 \pm 0.94$ & $0.51 \pm 0.04$ \\
\hline САMHB & $3.71 \pm 0.67$ & $3.66 \pm 0.24$ & $7.00 \pm 0.11$ & $0.17 \pm 0.02$ & $>14$ & $>49$ & $>55$ & $1.08 \pm 0.07$ \\
\hline LB & $1.76 \pm 0.06$ & $2.57 \pm 0.28$ & $3.91 \pm 0.33$ & $0.22 \pm 0.01$ & $>14$ & $20.6 \pm 1.54$ & $32.4 \pm 0.11$ & $0.76 \pm 0.08$ \\
\hline TSB & $14.0 \pm 1.70$ & $0.83 \pm 0.21$ & $3.96 \pm 0.22$ & $0.16 \pm 0.04$ & $>128$ & $18.0 \pm 5.74$ & $>256$ & $1.27 \pm 0.26$ \\
\hline RPMI & $>14$ & $3.64 \pm 0.39$ & $22.4 \pm 1.45$ & $0.20 \pm 0.02$ & $>14$ & $>49$ & $>55$ & $1.22 \pm 0.09$ \\
\hline
\end{tabular}

${ }^{a}$ The minimal inhibitory concentration (MIC) for selected antimicrobial peptides against E. coli NCTC 9001 or P. aeruginosa PAO1 is reported in micromolar for selected antimicrobial peptides as an average \pm standard error of three independent repeats. Buforin II was also tested in all media except for TSB. Only in LB against E. coli NCTC 9001 could an MIC $(26.6 \pm 0.9)$ be found as in all other conditions no inhibitory effect was observed. Values in bold are significantly different $(p<0.05)$ with respect to the corresponding MHB condition as determined by one-way ANOVA with Bonferroni post-hoc test.

perspective. The essential genome ${ }^{5}$ and transcriptome during infection $^{6-8}$ has been described for $P$. aeruginosa as has, more recently, the Staphylococcus aureus transcriptome during Cystic Fibrosis (CF) lung infection. ${ }^{9}$ Metabolomics has also been employed to understand how $P$. aeruginosa adapts to varying environments $^{10}$ and, in understanding its particular metabolic requirements during pulmonary infections, notably of $\mathrm{CF}$ patients, ${ }^{11}$ and this understanding is recognized both as being a route to more effective therapy ${ }^{9}$ and patient outcomes. ${ }^{12}$ These efforts have led to the development of complex, synthetic media that better reflect the nutritional environment in, for example, the CF lung infection setting. ${ }^{5,12,13}$ These may be further adapted to include components of the host innate immune system. ${ }^{7}$ In addition, ex vivo porcine lung models are being developed that recapitulate the spatial structure and nutritional environment of the host organ ${ }^{14,15}$ and which may better represent features of clinical disease. ${ }^{16}$

Establishing a mechanistic basis for bacterial species and/or strain-dependent environmental variation in antibiotic susceptibility will aid the development of chemically defined media that can more reliably predict therapeutic outcomes but also will contribute to rational antibiotic design. The present study aims to provide a mechanistic understanding of altered susceptibility to membrane active antibiotics, in particular to identify which components of bacterial and cell culture media have the greatest influence on cell wall structure. Since the production of most high energy compounds occurs at the bacterial plasma membrane, we hypothesize that altered metabolic strategy is likely to affect the composition of the bacterial cell envelope and may have a concomitant impact on susceptibility to antibiotics that act on these structures. We therefore chose to study the effect of culturing two Gram-negative bacteria, in a range of bacteriological media as well as RPMI-1640 (RPMI), on the metabolic strategy, the cellular metabolite composition and susceptibility to membrane active antibiotics. We use ${ }^{1} \mathrm{H}$ NMR of spent culture media to provide an overview of the metabolism of Escherichia coli NCTC 9001 and P. aeruginosa PAO1 in MHB, cation-adjusted MHB (CAMHB), Luria-Bertani (LB), Tryptone Soya Broth (TSB) and RPMI with 5\% fetal bovine serum (FBS), and ${ }^{1} \mathrm{H}$ high resolution magic angle spinning (HR-MAS) NMR of whole cells to describe changes in the cellular metabolite including the cell wall composition. The HR-MAS is supported by transmission electron microscopy (TEM) that shows how cell morphology is altered in the same conditions. Our study of E. coli NCTC 9001 is sensitive to fermentation and detects components of the previously well characterized acid stress response and this served to validate the usefulness of the NMR technique. Applying the same technique to P. aeruginosa, we characterize the metabolism of a panel of six isolates in MHB and RPMI and develop and test hypotheses regarding the effects of key components of bacteriological media and RPMI. Using a combination of both NMR methods and TEM, we show how the cell wall composition changes in response to the availability of formate, present in and consumed from all bacteriological media but largely absent from RPMI, and how this and other organic acids influence antibiotic sensitivity/tolerance.

\section{RESULTS}

Antibiotic Susceptibility of $E$. coli NCTC 9001 and $P$. aeruginosa PAO1 to Membrane Active Antimicrobials Is Strongly Influenced by Culture Conditions. To better understand how variations in metabolism and cell wall composition might impact on the potency of membrane active antimicrobials with differing mechanisms of action we selected four well-characterized antimicrobial peptides (AMPs) and assessed their potency in five different media against two representative Gram-negative bacterial isolates: uropathogenic E. coli NCTC 9001 and P. aeruginosa PAO1 (Table 1). LL-37 is a very well-studied human host defense peptide (HDP) with a multifaceted role in innate immunity. ${ }^{17-19}$ Magainin 2 is perhaps the archetypal membrane damaging AMP. ${ }^{20-22}$ Pleurocidin, generally more potent toward Gram-negative than Grampositive bacteria in $\mathrm{MHB},{ }^{23}$ is considered to have the ability to penetrate within bacteria as well as membrane damaging properties. $^{23-27}$ Colistin is a clinically relevant, polycationic cyclic polymyxin peptide considered the last-resort for multidrug-resistant Gram-negative infections. Although colistin is widely considered to act by disruption of the outer membrane integrity through displacement of $\mathrm{Mg}^{2+}$ and $\mathrm{Ca}^{2+}$ and binding to lipid $A,{ }^{28}$ polymyxins have also been suggested to insert into the plasma membrane, ${ }^{29}$ and fluorescently labeled analogues of polymyxin B have been shown to penetrate within Gramnegative bacterial cells. ${ }^{30}$ More recently, the discovery that MCR-1 mediated colistin resistance is dependent on the modification of cytoplasmic membrane associated lipopolysaccharide (LPS) and not that located at the outer membrane has enabled the demonstration that it is the targeting of cytoplasmic membrane bound LPS on which the bactericidal activity of colistin depends. ${ }^{31}$ Against either isolate, all four AMPs are most potent when tested in MHB. The potency of the linear AMPs (LL-37, pleurocidin, and magainin 2) is frequently substantially reduced both in the other three bacteriological media and RPMI. Although the effect is much more modest, the potency of colistin is also significantly reduced in the other media.

MHB is commonly adjusted with additional $\mathrm{Mg}^{2+}$ and $\mathrm{Ca}^{2+}$ to increase the concentration of each respectively from 228 and 73 

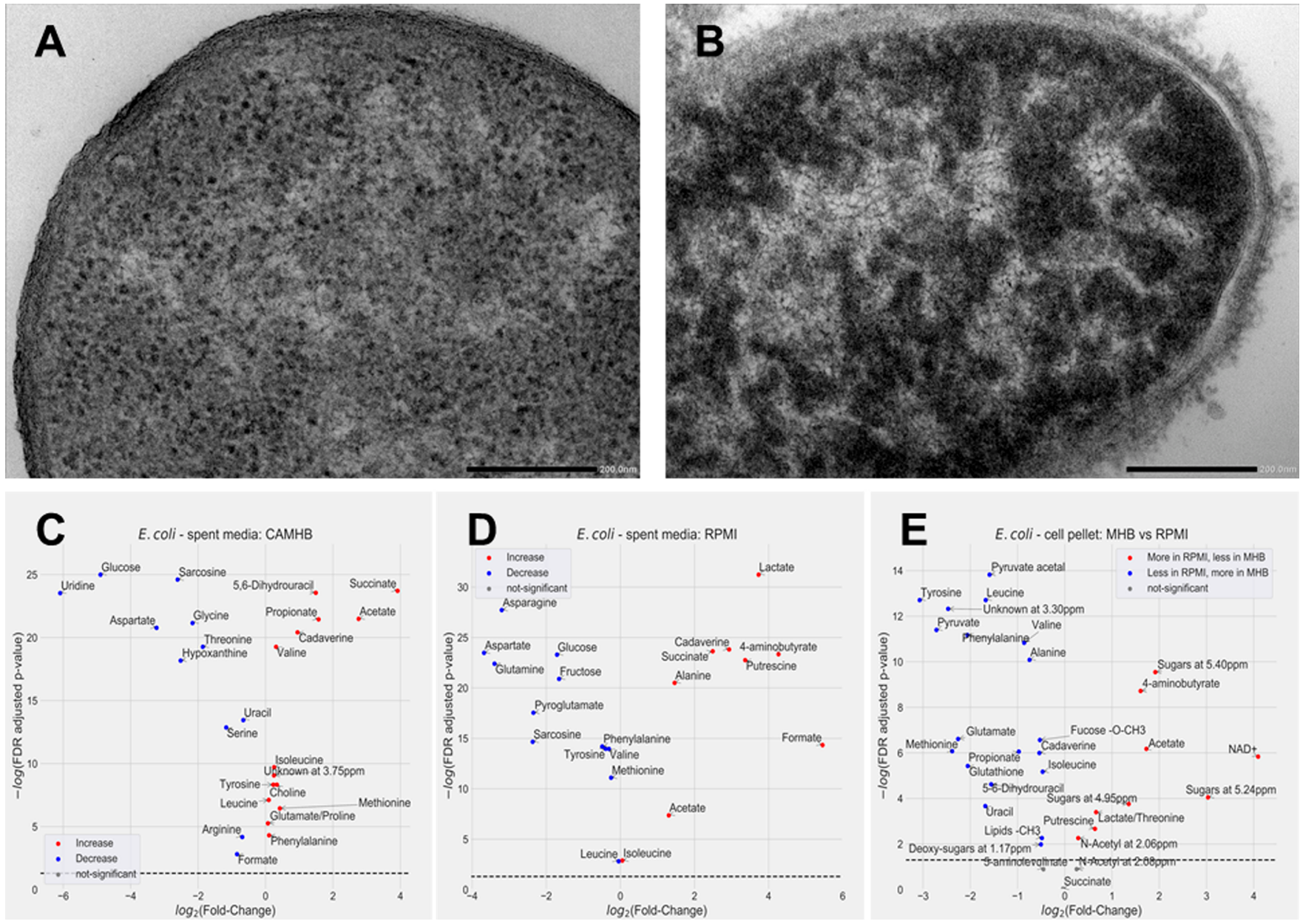

Figure 1. Glucose fermentation initiates an acid stress response in E. coli NCTC 9001. Transmission electron micrographs for E. coli NCTC9001 grown in MHB (A) or RPMI (B); wider field images are shown in Figure S2. Volcano plots obtained from liquid state ${ }^{1} \mathrm{H}$ NMR of spent bacterial culture reveal the metabolic strategy of E. coli NCTC 9001 in MHB (C) or RPMI (D). ${ }^{1} \mathrm{H}$ HR-MAS NMR of bacterial pellets enables comparison of the effect on cellular metabolites of growth in MHB or RPMI (E). Scale bars represent $200 \mathrm{~nm}$.

$\mu \mathrm{M}$ to 514 and $624 \mu \mathrm{M}$ in $\mathrm{CAMHB}$ and this is known to impact on the potency of AMPs. ${ }^{1,32}$ Cation adjustment affects the sensitivity of both isolates to the linear AMPs much more than to colistin. Both pleurocidin and magainin 2 experience a 4-fold reduction in sensitivity against $E$. coli NCTC 9001, and against $P$. aeruginosa PAO1 the potency is reduced below the limit of detection. The cation adjustment effect on LL-37 activity against E. coli NCTC 9001 is more moderate but activity against $P$. aeruginosa PAO1 is attenuated in the range of concentrations tested. In contrast, the activity of colistin toward E. coli NCTC 9001 is largely unaffected by cation supplementation. LB has a similar $\mathrm{Mg}^{2+}$ and $\mathrm{Ca}^{2+}$ composition to $\mathrm{MHB}$ before cation adjustment (Table S1). The reduction in potency of both pleurocidin and magainin 2 against both isolates, and LL-37 against $P$. aeruginosa $\mathrm{PAO} 1$, in this media is then due to other factors.

Colistin, though less sensitive to the use of differing media, is less effective in RPMI when compared with MHB where the MIC is approximately double. Except for the activity of pleurocidin against E. coli NCTC 9001, activity of the linear AMPs is drastically reduced in RPMI. The concentrations of $\mathrm{Mg}^{2+}(410 \mu \mathrm{M})$ and $\mathrm{Ca}^{2+}(420 \mu \mathrm{M})$ in RPMI are a little lower than in CAMHB but much greater than in either MHB or LB and can be expected to contribute to the reduced potency of the linear AMPs in the mammalian cell culture media. Additionally, a contribution from bacterial and/or serum proteases to neutralizing linear AMP activity can be expected; recently we compared pleurocidin and its D-amino acid analogue and found the D-enantiomer offered gains in potency, relative to the Lenantiomer, against a range of Gram-negative isolates in RPMI but not in MHB. ${ }^{23}$ Nevertheless, the observation that pleurocidin retains some useful activity in RPMI against E. coli NCTC 9001 but that this is not reproduced for P. aeruginosa PAO1 shows the effect of cation adjustment and protease activity are insufficient to explain the species specific susceptibility pattern. We therefore characterized metabolism in the microbiological media and RPMI to identify metabolic-dependent features of the bacterial envelope that may be associated with altered sensitivity to AMPs.

Altered Cell Wall Composition in E. coli NCTC 9001 Grown in RPMI Is Associated with an Acid Stress Response. A combination of transmission electron microscopy (TEM) (Figure 1A,B) and NMR spectroscopy (Figure 1C-E; Figure S1A-C) was used to study the response of E. coli NCTC 9001 to culture in four different bacteriological media or RPMI. Studying the spent culture metabolome suggests that a combination of fermentation and anaerobic respiration is employed in MHB (Figure 1). There is little glucose in MHB but this, together with serine and threonine, is consumed and fermented to acetate (see Figure 2 for pathway overview). 


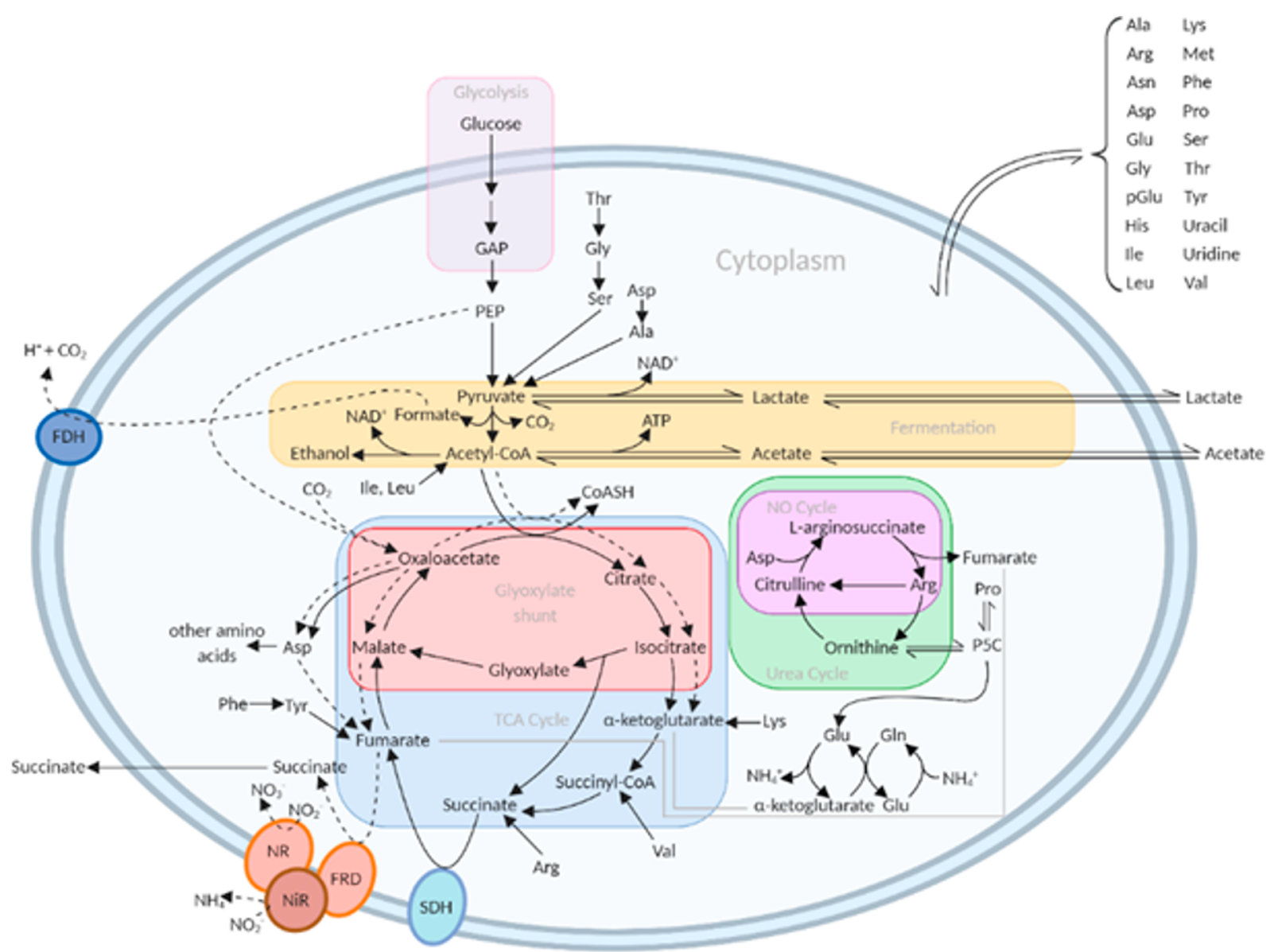

Figure 2. The major catabolic pathways available to E. coli or $P$. aeruginosa. Shown are key enzymes involved in anaerobic respiration: formate dehydrogenase $(\mathrm{FDH})$, succinate dehydrogenase $(\mathrm{SDH})$, nitrate reductase $(\mathrm{NR})$, nitrite reductase $(\mathrm{NiR})$.

Formate is an important electron donor for anaerobic respiration while nitrate, nitrite, and/or fumarate are common electron acceptors (Figure 2). The consumption of formate is therefore indicative of formate dehydrogenase activity while the consumption of aspartate and production of succinate are indicative of fumarate reductase activity (Figure 2) and taken together identify anaerobic respiration as an important feature of E. coli NCTC 9001 growth in MHB. A greater amount of glucose in TSB (2.5 g/Liter $13.9 \mathrm{mM}$ ), and the disaccharide trehalose in LB are linked to a slightly greater reliance on fermentation with production of formate and, for TSB only, lactate observed (Figure S1B/C). However, the consistent consumption of aspartate and excretion of succinate observed in CAMHB, LB, and TSB spent cultures (Figure $\mathrm{S} 1 \mathrm{~A}-\mathrm{C}$ ) indicates anaerobic respiration underpins growth of E. coli NCTC 9001 in all four bacteriological media. In contrast, both the NMR (Figure 1D) and, more simply, $\mathrm{pH}$ measurements of spent culture (Table 2) indicate that while anaerobic respiration continues, $E$. coli NCTC 9001 places much greater reliance on fermentation of glucose when growing in RPMI. Glucose is present at $2 \mathrm{~g} / \mathrm{L}$ $(11.1 \mathrm{mM})$ in RPMI and much of this is fermented to acetate, formate, and lactate. The result is that the culture is acidified by more than three $\mathrm{pH}$ units to around $\mathrm{pH} 4.6$, approximately two $\mathrm{pH}$ units lower than observed with the bacteriological media.

Enterobacteria including E. coli have stress responses that enable them to cope with mild and even severe low $\mathrm{pH}$ stress. ${ }^{33,34}$ Part of the acid stress response includes induction of amino acid decarboxylases which act to balance $\mathrm{pH}$ changes by
Table 2. $\mathrm{pH}$ of the Freshly Prepared Sterile Media and after Overnight Growth of E. coli NCTC 9001 or P. aeruginosa PAO1 $^{a}$

$\begin{array}{lccc} & \text { fresh } & \begin{array}{c}\text { E. coli NCTC } \\ 9001\end{array} & \begin{array}{c}\text { P. aeruginosa } \\ \text { PAO1 }\end{array} \\ \text { MHB } & 7.49 \pm 0.02 & 6.73 \pm 0.01 & 7.87 \pm 0.01 \\ \text { CAMHB } & 7.53 \pm 0.02 & 6.91 \pm 0.02 & 7.63 \pm 0.01 \\ \text { LB } & 7.28 \pm 0.01 & 7.31 \pm 0.01 & 7.76 \pm 0.01 \\ \text { TSB } & 7.30 \pm 0.02 & 6.44 \pm 0.02 & 7.46 \pm 0.07 \\ \text { RPMI } & 7.64 \pm 0.03 & 4.62 \pm 0.01 & 7.93 \pm 0.09 \\ \text { RPMI + 40 mM Na } & 7.32 \pm 0.02 & \text { ND }^{b} & 7.95 \pm 0.09\end{array}$

${ }^{a}$ Data are from the overnight cultures used for the NMR metabolomics experiments, described in Figures 2 or 3, presented as averages \pm standard error of mean. ${ }^{b} \mathrm{ND}=$ not done.

consuming a proton during decarboxylation and then exchange the product for a new substrate via a membrane bound antiporter. In this way, glutamate is exchanged for GABA (4aminobutyrate), arginine for agmatine, lysine for cadaverine, and ornithine for putrescine. Increased putrescine, cadaverine, and 4-aminobutyrate are detected in spent RPMI culture while substantial amounts of glutamine, present at $300 \mathrm{mg} / \mathrm{L}$, are also consumed (Figure 1D). Glutamine can itself be decarboxylated or it can be deaminated via an acid-activated glutaminase, $\mathrm{YbaS}$, releasing free ammonia which neutralizes the proton and yielding glutamate in the cell as a substrate for decarboxylation. 

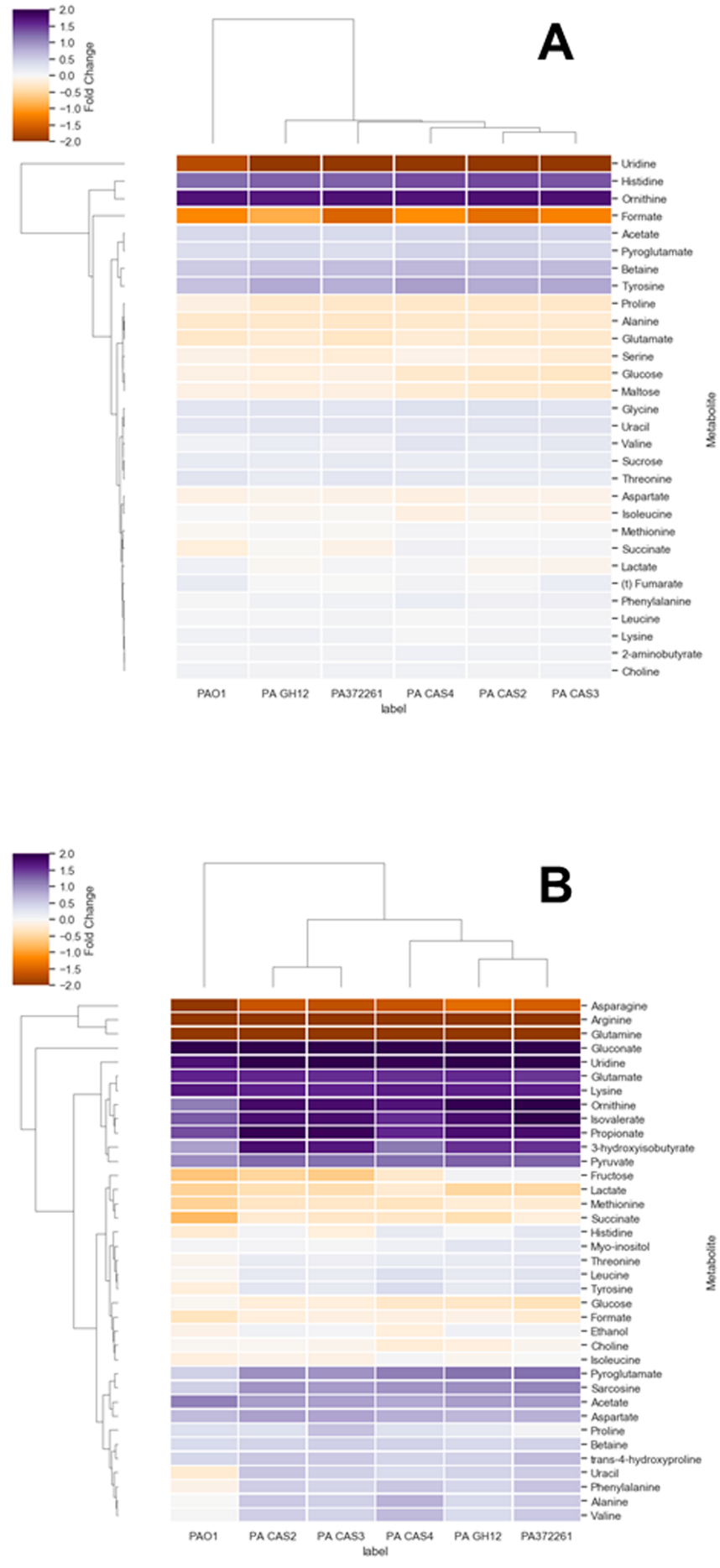
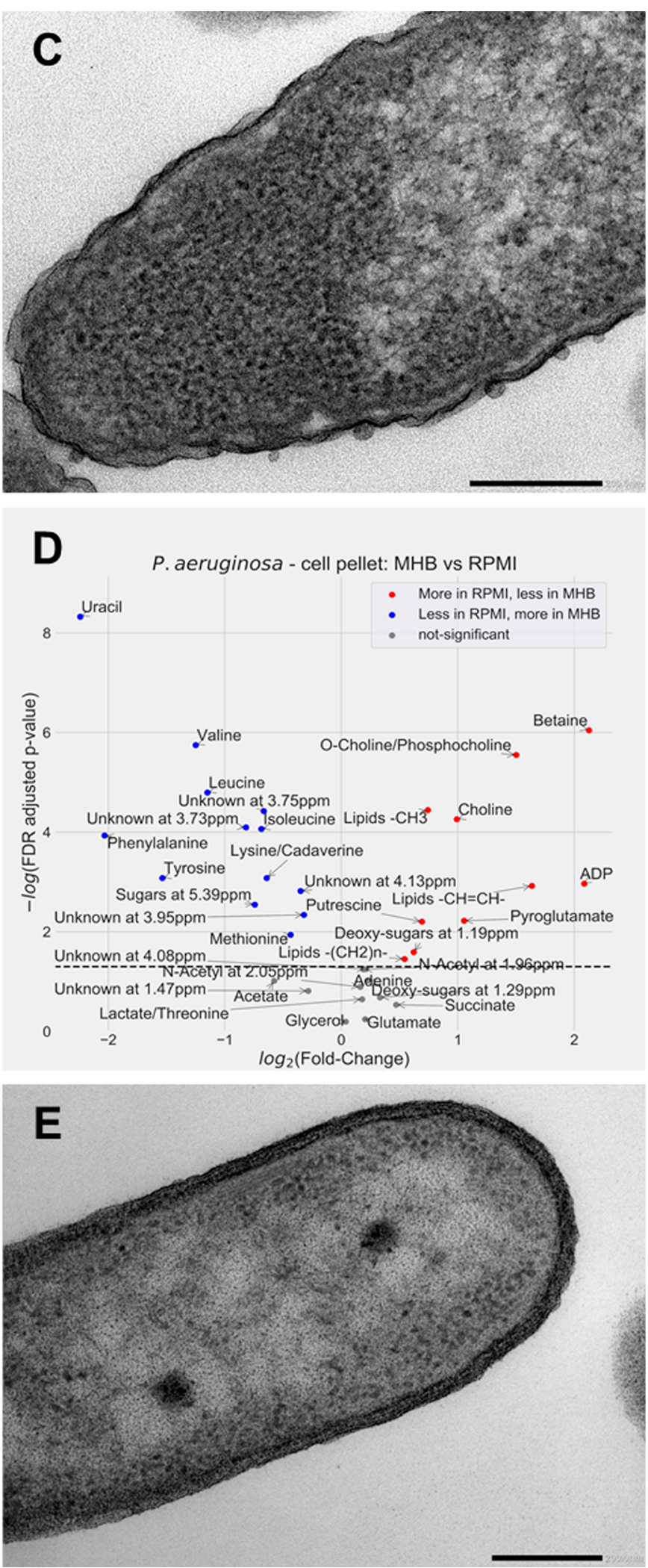

Figure 3. Anaerobic respiration and osmotic stress affect cell wall composition in P. aeruginosa PAO1. Hierarchical cluster maps obtained from liquid state ${ }^{1} \mathrm{H}$ NMR of spent bacterial culture reveal the metabolic strategy of $P$. aeruginosa in MHB (A) or RPMI (B). Transmission electron micrographs for P. aeruginosa PAO1 grown in MHB (C) or RPMI (E); wider field images are shown in Figure S2. Scale bars represent $200 \mathrm{~nm}$. ${ }^{1} \mathrm{H}$ HR-MAS NMR of P. aeruginosa PAO1 bacterial pellets enables comparison of the effect on cellular metabolites of growth in MHB or RPMI (D).

The glutamate-dependent acid resistance (AR) system, AR2, is regulated by the DNA-binding transcriptional activator GadE. $^{36}$ GadE is itself activated during exponential phase growth at mildly acidic $\mathrm{pH}$ via the two-component system (TCS) EvgAS which also activates a second TCS, PhoPQ via the connector SafA. Consistent with this, spent RPMI culture 

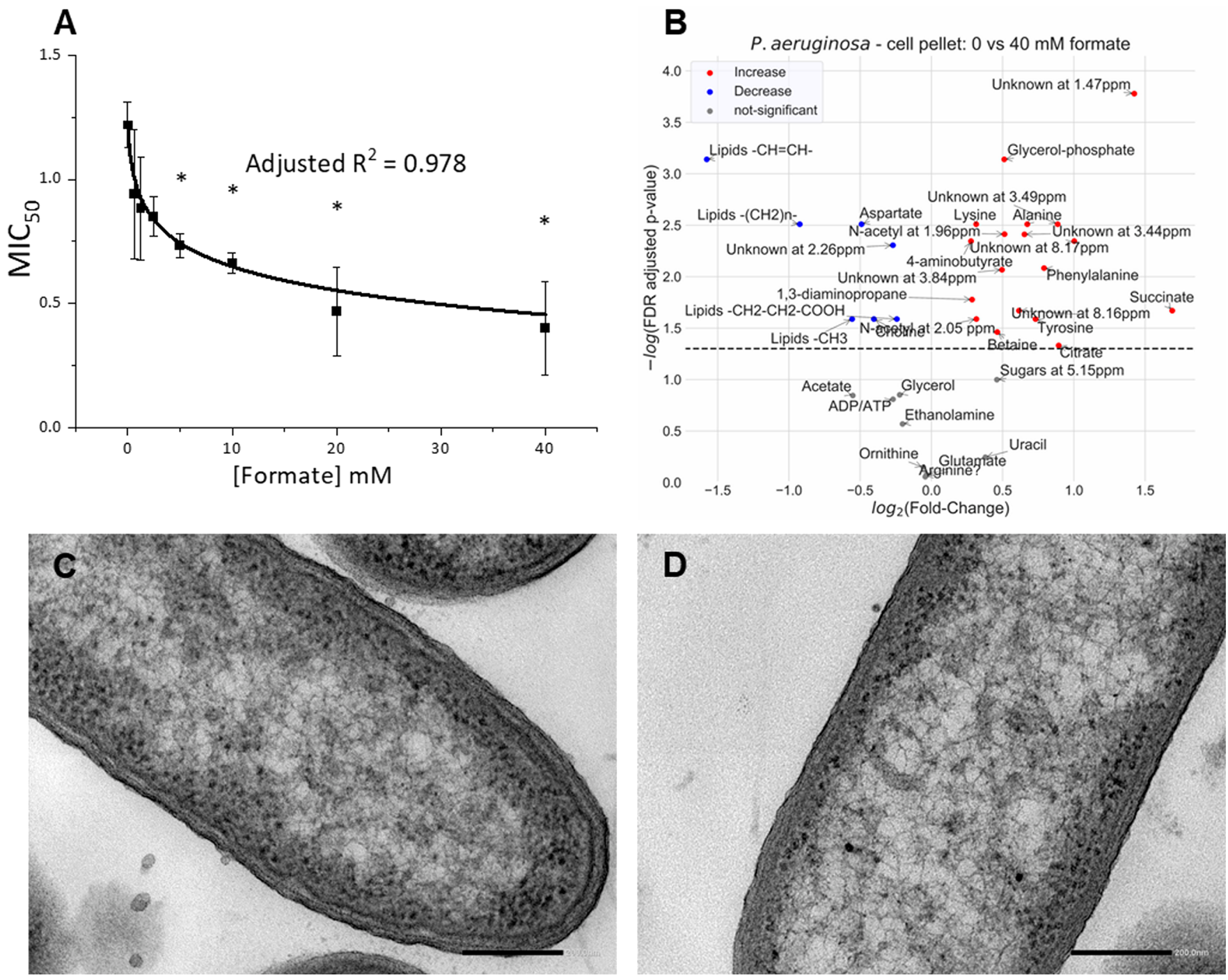

Figure 4. Formate doping of RPMI increases sensitivity of $P$. aeruginosa PAO1 to colistin and induces cell wall remodelling. The colistin MIC for $P$. aeruginosa PAO1 in RPMI decreases as a function of added formate (A). In a broth-microdilution assay, at $5 \mathrm{mM}$ [formate] and above significant reductions in the MIC are observed ( $p<0.05$, one-way ANOVA with Bonferroni posthoc test) with an approximately 2 -fold reduction in MIC at 40 mM. ${ }^{1} \mathrm{H}$ HR-MAS NMR of bacterial pellets (B) or transmission electron microscopy (C,D) enables comparison of the effect on cellular metabolites and cell ultrastructure of growth in RPMI with (D) or without (C) $40 \mathrm{mM}$ formate. Scale bars represent $200 \mathrm{~nm}$.

obtained from growth of the $\Delta e v g S$ mutant of E. coli BW25113, obtained from the Keio collection, does not undergo the same level of acidification as the parent (Table S2). PhoPQ is known to regulate the sensitivity of $E$. coli to antimicrobial peptides by controlling EptB, which adds a phosphoethanolamine moiety, and $\mathrm{PagL}$ and $\mathrm{PagP}$, which respectively deacylate and acylate lipid $\mathrm{A}^{37,38}$

The production of the exopolysaccharide, colanic acid, or Mantigen, a negatively charged polymer of glucose, galactose, fucose, and glucuronic acid that forms a protective capsule, by UDP-glucose 6-dehydrogenase is controlled by RcsB, which is required for acid resistance and interacts with GadE. ${ }^{39}$ Consistent with this, HR-MAS NMR of whole cells shows that growth of E. coli NCTC 9001 in RPMI causes substantial changes in the cell wall composition relative to that found when grown in MHB and specifically increases in the intensity of three resonances assigned to sugar moieties and an $\mathrm{N}$-acetyl group (Figure 1E). In support of this finding, TEM images (Figure $1 \mathrm{~A}, \mathrm{~B}$; Figure S2A,B) reveal there is a clear change in the outer membrane structure (Figure 1A/B). In contrast, analogous comparisons of HR-MAS spectra obtained for E. coli NCTC 9001 grown in LB, TSB, or CAMHB (Figure S3A-C) show the cellular metabolite complement is altered substantially in each case but the major changes attributed to polysaccharide resonances are lacking. The conformation of LL-37 is $\mathrm{pH}$ dependent with the $\alpha$-helix conformation and antibacterial activity lost below $\mathrm{pH} 5 .{ }^{17,40}$ While the acidification of the spent media in TSB is modest (Table 2), this has nevertheless been shown to be sufficient to have a measurable impact on LL-37 activity against both $S$. aureus and $P$. aeruginosa. ${ }^{40}$ Therefore, the activity of LL-37 is likely to be impaired by environmental acidification in TSB and, more strongly, in RPMI. However, while magainin 2 is only approximately 2 -fold less active in TSB than in MHB it is nearly 13 times less potent in RPMI. The activity of magainin 2 is not known to be inhibited by low $\mathrm{pH}$; indeed, its anticancer activity is enhanced, ${ }^{41}$ and hence the reduction in its activity in RPMI is likely associated with the acid-stress response of the bacteria and the production of an additional polysaccharide barrier rather than any direct effect of $\mathrm{pH}$ on behavior of the peptide. 

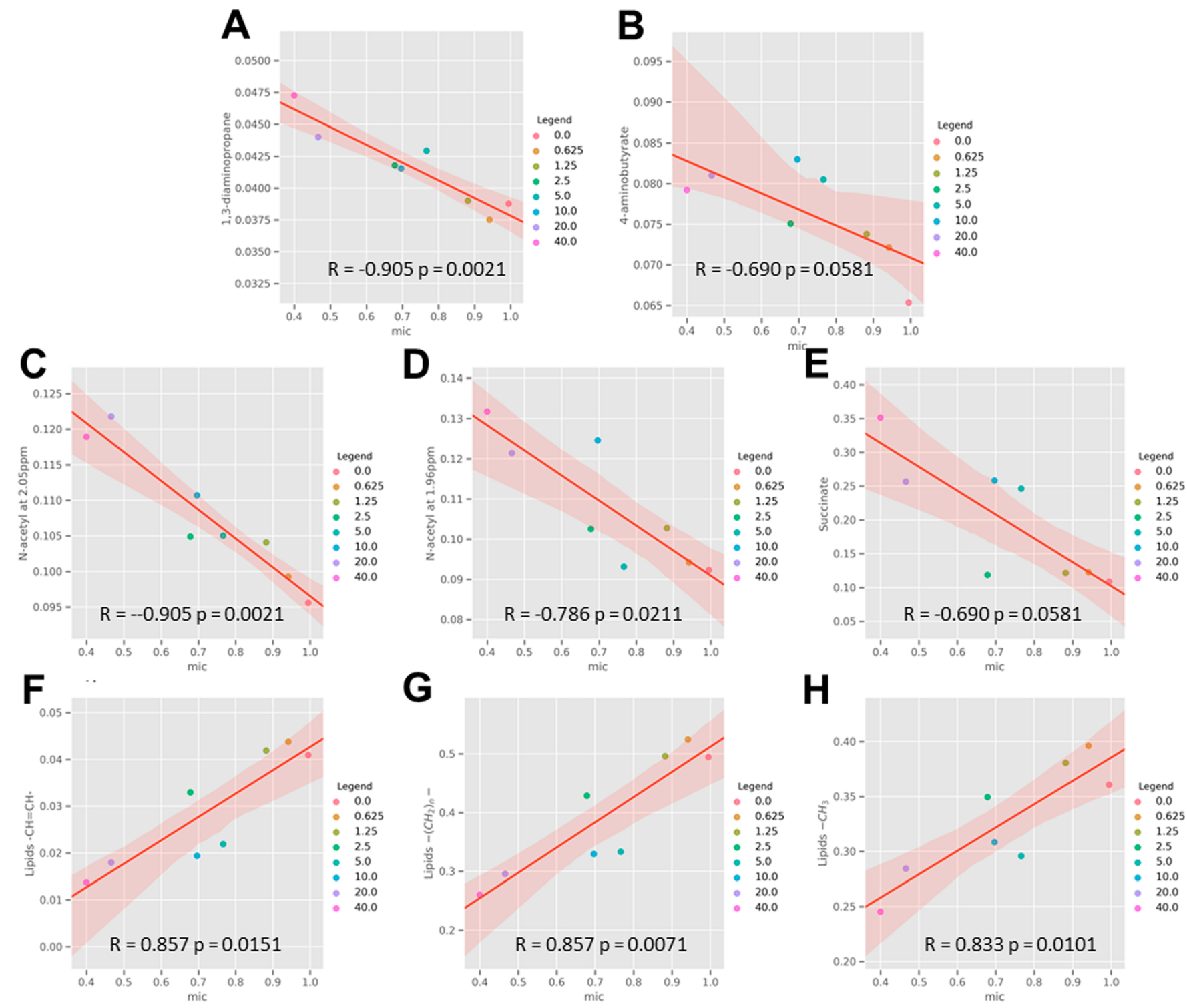

Figure 5. Colistin sensitivity induced by formate doping of RPMI is associated with polyamine catabolism and cell wall remodelling in $P$. aeruginosa PAO1. Linear regression analysis, of HR-MAS NMR data with MICs obtained from a separate broth microdilution assay, identifies metabolites (A-H) associated with increased sensitivity to colistin following addition of formate at the concentrations ( $\mathrm{mM}$ ) indicated in the legend for each panel.

\begin{abstract}
Altered Cell Wall Composition in P. aeruginosa PAO1 Grown in RPMI Is Associated with Osmotic Stress and Glucose and Formate Availability. NMR spectroscopy was also used to investigate the metabolic strategy of six multidrugresistant and susceptible clinical $P$. aeruginosa isolates in MHB or RPMI, and TEM and HR-MAS NMR were used, as above, to characterize P. aeruginosa PAO1 (Figure 3; Figure S1D-F). It is known that, unlike $E$. coli, $P$. aeruginosa eschews glucose consumption. ${ }^{42}$ In RPMI, some glucose is consumed, and gluconate and a little acetate is detected in the spent media (Figure 3B). It is therefore probable that some glucose in RPMI is being used via the Entner-Doudoroff pathway to support pyruvate fermentation, ${ }^{43}$ but the consumption of succinate, whose presence would repress the expression of genes needed for assimilation of sugars including gluconate, will limit this. ${ }^{42}$ Therefore, although the preferred metabolic strategy for $P$. aeruginosa may be aerobic respiration, and this may contribute to growth in the conditions used here, a more substantial role for anaerobic processes is expected. ${ }^{44}$ These would include both use
\end{abstract}

of arginine as an energy source via the arginine deiminase pathway and anaerobic respiration using a variety of electron donor and acceptors (Figure 2). Consistent with this, arginine is consumed, and ornithine excreted by $P$. aeruginosa PAO1 in all five media conditions and by all six $P$. aeruginosa isolates in both MHB and RPMI (Figure 3A,B; Figure S1D-F). Arginine consumption may be less in RPMI where glucose may exert catabolite repression. ${ }^{45}$ In contrast, while formate is proportionally the most consumed metabolite in all four bacteriological media (MHB, CAMHB, LB, and TSB), it is largely absent from RPMI and hence unavailable as a major substrate (Figure 3B).

Formate dehydrogenase-dependent anaerobic respiration is therefore identified as a key source of energy for $P$. aeruginosa when grown in the four bacteriological media but not RPMI where growth is instead underpinned by glutamine, asparagine, and lactate consumption (Figure 3B). P. aeruginosa does not grow well in RPMI until 5\% FBS is added from which lactate is consumed (Figure 3B). Lactate is an alternative electron donor for anaerobic respiration and hence growth of $P$. aeruginosa in 
Table 3. Effect of Different Media and Organic Acids on Susceptibility of a Panel of P. aeruginosa Isolates to Tobramycin or Colistin $^{a}$

\begin{tabular}{|c|c|c|c|c|c|c|c|c|}
\hline \multirow[b]{2}{*}{ P. aeruginosa isolate } & \multirow[b]{2}{*}{ TSB } & \multirow[b]{2}{*}{ МHB } & \multirow[b]{2}{*}{ RPMI } & \multicolumn{5}{|c|}{ RPMI $+40 \mathrm{mM}$ organic acid } \\
\hline & & & & Formate & Butyrate & Acetate & Citrate & Propionate \\
\hline \multicolumn{9}{|c|}{ Tobramycin } \\
\hline 372261 & 2 & 2 & 2 & 1 & 1 & 1 & 1 & 1 \\
\hline CAS2 & 4 & 4 & 4 & 2 & 2 & 2 & 1 & 2 \\
\hline CAS3 & 4 & 2 & 4 & 2 & 2 & 1 & 1 & 1 \\
\hline CAS4 & 4 & 3 & 4 & 4 & 2 & 2 & 2 & 2 \\
\hline GH12 & $>128$ & $>128$ & $>128$ & $>128$ & $>128$ & $>128$ & $>128$ & $>128$ \\
\hline PAO1 & 2 & 2 & 4 & 2 & 2 & 1 & 1 & 2 \\
\hline Average* $^{*} \pm \mathrm{SE}$ & $3.2 \pm 0.5$ & $2.8 \pm 0.5$ & $3.6 \pm 0.4$ & $2.2 \pm 0.5$ & $1.8 \pm 0.2$ & $1.4 \pm 0.2$ & $1.2 \pm 0.2$ & $1.6 \pm 0.2$ \\
\hline \multicolumn{9}{|c|}{ Colistin } \\
\hline 372261 & 1 & 2 & 2 & 1 & 2 & 1 & 16 & 1 \\
\hline CAS2 & 1 & 4 & 2 & 2 & 1 & 16 & 8 & 1 \\
\hline CAS3 & 0.5 & 2 & 1 & 1 & 1 & 1 & 1 & 0.5 \\
\hline CAS4 & 1 & 4 & 2 & 1 & 2 & 1 & 1 & 1 \\
\hline GH12 & 0.5 & 4 & 2 & 2 & 2 & 2 & 2 & 1 \\
\hline PAO1 & 1 & 1 & 2 & 1 & 1 & 2 & 1 & 1 \\
\hline Average $\pm S E$ & $0.8 \pm 0.1$ & $2.8 \pm 0.5$ & $1.8 \pm 0.2$ & $1.3 \pm 0.2$ & $1.5 \pm 0.2$ & $3.8 \pm 2.4$ & $4.8 \pm 2.5$ & $0.9 \pm 0.1$ \\
\hline
\end{tabular}

${ }^{a}$ Concordant MICs are provided from three independently repeated experiments. For sensitive strains only, an average MIC is provided in each condition and significant differences relative to RPMI, as determined by One-way ANOVA with Geisser-Greenhouse correction and Dunnett's multiple comparisons test, in bold. RPMI conditions all contain 5\% FBS.

RPMI is likely supported by anaerobic respiration and arginine catabolism with glutamine and asparagine as major sources of ammonium.

Despite the similarities in growth strategies used by $P$. aeruginosa PAO1 in MHB and RPMI, HR-MAS NMR analysis again detects substantial differences in the cell wall composition for cells grown in the two media. Specifically, resonances assigned to lipids and the osmoprotectant glycine betaine ${ }^{46}$ are substantially increased in cells grown in RPMI (Figure 3D). These findings are supported by the TEM images since cells grown in RPMI appear more turgid and the cell wall appears thicker and is much more densely stained (Figure 3C,E; Figure S2). More modest changes, relative to $\mathrm{MHB}$, were detected by HR-MAS NMR for cells grown in LB or TSB though there is a notable increase in glycine betaine for the latter (Figure S3D,E). Consistent with this, cells grown in TSB appear more turgid but there is little change in the thickness or staining of the cell wall (Figure S4). Consequently, in addition to characterizing the effect of increased $\mathrm{Mg}^{2+}$ and $\mathrm{Ca}^{2+}$ on the cell wall in $P$. aeruginosa PAO1 (Figure S5) we investigated the effect of glucose addition to MHB (Figure S6), and formate addition to RPMI (Figure 4/ $5)$, to isolate the contributions of these nutrients to the different phenotypes observed in MHB and RPMI. Titration of $\mathrm{Ca}^{2+}$ and $\mathrm{Mg}^{2+}$ in MHB to levels that match and then surpass those found in RPMI (though a little lower than in CAMHB) induces little change in the susceptibility of $P$. aeruginosa PAO1 to colistin (Figure S5B).

HR-MAS NMR nevertheless identifies substantial changes in the cellular metabolite complement including increased intensity of resonances assigned to lipids (Figure S5D). In contrast, addition of glucose to MHB was associated with a modest increase in tolerance to colistin and an increase in the osmoprotectant glycine betaine but no effect on cell wall components (Figures S6 and S7).

Increased Sensitivity of $P$. aeruginosa to Membrane Active Antimicrobials Is a Nonspecific Effect of Doping with Organic Acids. When formate is added to RPMI, very little is consumed but its addition has profound effects on cell wall composition in $P$. aeruginosa PAO1 and is strongly correlated with a modestly increased susceptibility to colistin (Figure 4) despite a relatively modest impact on the overall metabolic strategy (Figures S8 and S9). Addition of formate correlates with an approximate two-fold decrease in colistin sensitivity and the relationship is best described by a logarithmic transform (Figure 4A). Low doses of formate to RPMI, a final concentration of $10 \mathrm{mM}$ or less, caused only modest changes in metabolites excreted or consumed by $P$. aeruginosa PAO1 (Figures S8 and 9). At $20 \mathrm{mM}$ [formate] and above, these changes become more substantial (Figure S8) but the metabolic strategy is not fundamentally changed (Figure S9). Increased production of propionate and 3-hydroxyisobutyrate is notable as is increased consumption of choline and amino acids including histidine, isoleucine, leucine, methionine, phenylalanine, threonine, tyrosine, and valine. Production of aspartate, glutamate, pyruvate, and lysine is halted and production of ornithine decreases. However, the large-scale consumption of arginine and glutamine is unaffected, increases in lactate consumption are modest, and there is no effect on succinate consumption. Consistent with this, no significant effect of formate addition can be observed in DCFH-DA fluorescence as a measure of ROS/RNS generation and a possible indicator of nitrate or nitrite reductase activity; this is modestly if significantly lower in RPMI compared with MHB (Figure S8D).

In contrast with the liquid state $\mathrm{NMR}$, at $5 \mathrm{mM}$ [formate] and above, HR-MAS NMR of cells grown in RPMI with and without formate detects substantial reductions in the intensity of resonances assigned to lipids (Figure 4B). Consistent with this, the corresponding TEM of $P$. aeruginosa PAO1, grown in RPMI with and without $40 \mathrm{mM}$ [formate], shows the cells to be similarly turgid but the staining of the inner and outer membrane is noticeably denser in the absence of formate (Figure 4C,D). Additionally, HR-MAS identifies reduced cellular aspartate and increased succinate, 1,3-diaminopropane, and 4-aminobutyrate (also known as $\gamma$-aminobutyric acid or GABA). These last two metabolites are both associated with spermine and spermidine catabolism and hence polyamine 
degradation. GABA is also a product of putrescine degradation and can be further degraded to succinate. ${ }^{47}$

Although no MIC could be determined for the three linear AMPs in RPMI, this data was available for colistin and could also be determined, at various formate concentrations, in a separate broth microdilution assay. PLS regression between the HR-MAS NMR metabolomic data and MIC as a function of formate concentration (Figure 5) and Spearman correlations were calculated between all metabolites quantified in the formate titration experiment (Figure S10). A good model was obtained $\left(Q^{2}=0.370 ; R^{2}=0.944\right.$; Figure S11) with the variance of 11 metabolites correlating $(p<0.05)$ with the average MIC (Figure 5 ; Figure S11). The strongest correlation with the decreasing MIC is obtained with 1,3-diaminopropane, lysine, and a resonance assigned to an $\mathrm{N}$-acetyl group (Figure $5 \mathrm{~A}, \mathrm{C}$ ). A similar trend is also observed for GABA (Figure $5 \mathrm{~B} ; p=0.0581$ ). Weaker correlations are observed with lipid resonances (Figure $5 \mathrm{~F}-\mathrm{H}$ ), succinate (Figure $5 \mathrm{E}$ ) and aspartate, alanine, phenylalanine and acetate (Figure S11). The correlation between the colistin MIC and betaine is relatively weak $(p=0.0861)$. The decreased tolerance to colistin with increasing formate concentration is therefore most closely associated with polyamine degradation though the remodelling of the plasma membrane may also contribute.

Since the formate-dependent sensitization of $P$. aeruginosa PAO1 to colistin was achieved in the absence of a fundamental change in metabolism we hypothesized that this might be a generic effect, achievable with other organic acids, that might impact the activity of other membrane active antimicrobials. We therefore obtained MICs for both colistin and the aminoglycoside tobramycin for the six $P$. aeruginosa isolates in MHB, TSB, and RPMI (5\% FBS) alone or containing $40 \mathrm{mM}$ formate, butyrate, acetate, citrate, or propionate (Table 3). GH12 is resistant to tobramycin $(\mathrm{MIC}>128 \mu \mathrm{g} / \mathrm{mL})$ but the five other strains are sensitive and the MICs in RPMI are consistently reduced through the addition of the five organic acids. Two-fold reductions in MIC are observed in all but one instance and fourfold reductions observed in the presence of acetate, citrate and propionate. The impact of the five organic acids on colistin MICs are more variable and, when potentiating, more modest. Substantial increases in colistin MIC are observed for two strains (372261 and CAS2) in the presence of citrate, while acetate produces the same effect for CAS2 only. Notably, however, propionate consistently induces a 2 -fold reduction in colistin MIC.

\section{DISCUSSION}

The present study shows that the combination of both liquid and HR-MAS NMR spectroscopy, supported by TEM, is an effective means of characterizing the metabolic strategies of bacteria in varying growth conditions and can be used to identify contributions of individual nutrients to antimicrobial susceptibility phenotypes. The acid stress response of $E$. coli has been described in some depth previously and its characterization for E. coli NCTC 9001 in glucose rich (11.1 mM) RPMI, serves here to validate the approach and to indicate that, in glucose rich infection settings, we may expect AMPs to face substantial amounts of colanic acid as an additional barrier. Since the acid stress response is more muted in similarly glucose rich TSB $(13.9 \mathrm{mM})$, its fermentation and consequent acidification of the environment will depend on the availability of other nutrients. In urine, glucose concentrations are normally low, less than $0.7 \mathrm{mM}$ in healthy subjects but can exceed $2.5 \mathrm{mM}$ in patients with diabetes or kidney disease. ${ }^{48}$ In blood, glucose is normally available in the low millimolar range ( 3.9 to $5.8 \mathrm{mM})$ when fasting but exceeds $7 \mathrm{mM}$ in diabetics. ${ }^{49}$ Therefore, since exposure of E. coli to sublethal doses of AMPs is known to trigger production of colanic acid (and curli) and such priming confers tolerance and persistence, ${ }^{50}$ in vitro susceptibility testing, capable of predicting in vivo success against E. coli by AMPs in such settings, may therefore incorporate glucose to trigger the production of this barrier.

The NMR metabolomic approach was also capable of describing the metabolic strategy of $P$. aeruginosa PAO1 in the same varying growth conditions, additionally isolating the role of three key nutrients, a combination of the divalent cations $\mathrm{Ca}^{2+}$ and $\mathrm{Mg}^{2+}$, glucose, and formate, in determining the composition of the cell wall and susceptibility to AMPs including colistin. The relative importance of each of these for susceptibility testing of $P$. aeruginosa can therefore be considered.

Cation adjustment of MHB is known to inhibit the activity of AMPs. ${ }^{33}$ Here, the addition of divalent cations to MHB affected the activity of linear AMPs but had minimal impact on the activity of colistin. The reduction of activity of the linear AMPs against $P$. aeruginosa $\mathrm{PAO} 1$ can be attributed to changes in, predominantly, lipids which is consistent with the requirement for the plasma membrane to be damaged or crossed as the key element of their mechanisms of action. Consequently, it might be appropriate to recommend cation adjustment if this were to accurately model the in vivo conditions. However, cation adjustment alone is unlikely to predict in vivo activity for both $E$. coli and $P$. aeruginosa since the effect of cation adjustment on $P$. aeruginosa is more profound than on E. coli and it does not reproduce the same reduction in potency for LL-37 or magainin 2 against E. coli NCTC 9001 as found in RPMI, which may be related to the acid stress response discussed above. Addition of $\mathrm{Ca}^{2+}$ and $\mathrm{Mg}^{2+}$ to $\mathrm{MHB}$ up to and beyond the levels found in RPMI did not significantly increase the MIC of colistin against $P$. aeruginosa PAO1. Only at the highest $\left[\mathrm{Ca}^{2+}\right]$ and $\left[\mathrm{Mg}^{2+}\right]$ found in CAMHB was a significant, if modest, increase observed. This is despite substantial changes in the plasma membrane lipid composition and even signals that may be attributed to LPS and/or EPS. If penetration of the plasma membrane by colistin contributes substantially to its bactericidal action, ${ }^{29,30}$ then the observed changes in cellular metabolite composition might be expected to have more impact. However, the recent discovery that colistin targets cytoplasmic membrane bound LPS puts these findings in new light since they imply that cytoplasmic membrane bound LPS may not be affected in the same way as outer membrane bound LPS, reducing the sensitivity of colistin to changes in environmental $\mathrm{Ca}^{2+}$ and $\mathrm{Mg}^{2+}$.

Glucose Availability Will Influence Osmotic Potential. Glucose is considered to be available in millimolar concentrations in CF sputum. ${ }^{51}$ However, although it may be an important carbon source for some species, for $P$. aeruginosa glucose is considered a secondary carbon source. ${ }^{8}$ Instead, its preferred carbon sources are proline, alanine, arginine, lactate, glutamate, and aspartate, ${ }^{51}$ and expression of $P$. aeruginosa lactate dehydrogenase is increased in vivo in cystic fibrosis lungs. ${ }^{7,8}$ In the present study, arginine and lactate are all consumed from RPMI by the six $P$. aeruginosa isolates in addition to, most notably, glutamine and asparagine. Glutamine $(2 \mathrm{mM})$ and asparagine $(0.38 \mathrm{mM})$ are likely important sources of ammonium in RPMI and can be compared with the $2.3 \mathrm{mM}$ $\mathrm{NH}_{4}{ }^{+}$available in synthetic cystic fibrosis medium (SCFM). ${ }^{51}$ Their catabolism likely explains the excretion of aspartate and 
glutamate into the spent media. Succinate, consumed by $P$. aeruginosa $\mathrm{PAO} 1$ and, to a lesser extent, by the other five isolates, is an important intermediate in the tricarboxylic acid (TCA) cycle but also the glyoxylate shunt. The latter enables $P$. aeruginosa to bypass the complete TCA cycle, in particular its $\mathrm{CO}_{2}$ generating steps, conserve carbon for gluconeogenesis, and reduce electron flow into respiration. ${ }^{52}$ Supplementing the shunt would aid in overcoming oxidative stress. ${ }^{52}$ RPMI also contains $1.2 \mathrm{mM} \mathrm{NO}_{2}{ }^{-}$, available from $100 \mathrm{mg} / \mathrm{Liter} \mathrm{Ca}\left(\mathrm{NO}_{3}\right)_{2}$, and hence the consumption of lactate as an electron donor is consistent with anaerobic respiration and denitrification as characterized in sputum samples. ${ }^{8}$ Therefore, although there are differences in the composition of RPMI and media designed to better reflect the CF infection setting, the overall metabolic strategy of $P$. aeruginosa in these conditions and in sputum is similar. Glucose availability thus does not have a substantial effect on $P$. aeruginosa metabolism beyond an apparent osmotic effect associated with the production of glycine-betaine and a more turgid appearance in TEM.

Sensitization of $P$. aeruginosa to Colistin or Tobramycin by Formate and Other Organic Acids. Addition of formate to RPMI has a profound effect on the lipid composition of $P$. aeruginosa PAO1 and modestly reduces its tolerance of colistin. The effect on the metabolic strategy is however not so profound and other organic acids can produce the same, modest sensitization of $P$. aeruginosa to colistin or the aminoglycoside tobramycin. The availability of formate or other organic acids in RPMI may therefore have a nonspecific effect and consideration of the nature of the changes in metabolites that are detected and that correlate with formate-induced colistin sensitivity support this. Significant changes in the cell metabolite complement occurred at $5 \mathrm{mM}$ [formate] and this coincides with significant reduction in tolerance to colistin. Increased succinate is identified intracellularly but there are no substantial changes in its consumption from the media and its intracellular concentration correlated only weakly with the MIC. This is perhaps because increased intracellular succinate could have multiple causes: (1) redirection of arginine metabolism from the arginine iminase to the arginine succinyltransferase (AST) pathway; (2) from anaerobic respiration where fumarate, produced from aspartate, acts as an electron acceptor; and (3) from spermine, spermidine, and/or putrescine degradation (via GABA). There is therefore evidence for a modest change in anaerobic respiration, that is, cellular aspartate is depleted and succinate increased, which may contribute to the observed change in MIC. However, the strongest correlation with the reduced MIC is with 1,3-diaminopropane and an $\mathrm{N}$-acetyl resonance. Whether through stimulation of anaerobic respiration or, more likely, an uncharacterized, nonspecific direct effect, formate addition stimulates polyamine degradation. In $P$. aeruginosa, polyamines are responsible for resistance to cationic peptides, aminoglycosides, and quinolone antibiotics ${ }^{53}$ but are also protective from oxidative stress when localized at the cell surface. ${ }^{54}$ Genes required for the production of polyamines are also transcribed under the control of the PmrAB twocomponent system, associated with resistance to both colistin and aminoglycosides, ${ }^{55}$ and spermidine synthase is part of the same gene operon. ${ }^{56}$ There may therefore be a link between oxidative stress, the need for polyamine synthesis, and concomitant reduced sensitivity to cationic antimicrobials but we did not see any mitigation of ROS on addition of formate, using the DCFH-DA fluorescent probe.
Because formate and other organic acids may be responsible for modestly increased sensitivity to cationic antimicrobials in vitro, there are now questions as to how this is achieved, whether their addition might be a useful modification to susceptibility testing media, and whether it has any relevance to the in vivo infection setting?

Organic acids are capable of crossing the bacterial plasma membrane, being protonated or deprotonated but also complexing with transition metals. Organic acids have been shown to shuttle transition metals into bacteria and this has been exploited to inhibit the growth of bacterial pathogens. ${ }^{57}$ Such activity may deplete extracellular and surface associated zinc, nickel and iron while enriching these within the bacteria. Future work may therefore explore whether and how the wide-ranging effects of this process influences the production of polyamines by $P$. aeruginosa or otherwise influences the activity of membrane active antimicrobials.

Consistent with the lack of a substantial shift in metabolic strategy on the addition of formate to RPMI in the present study, formate dehydrogenase encoding genes are found to be repressed in $P$. aeruginosa from $\mathrm{CF}$ sputum, ${ }^{8}$ and hence formate is therefore probably not a key electron donor in the pulmonary lung infection setting. There is however ample evidence from NMR studies that formate and acetate are present in substantial quantities in the lung, ${ }^{58-61}$ while propionate liberated by mucin fermenting anaerobes may support growth of $P$. aeruginosa in CF sputum. ${ }^{61}$ This study therefore suggests the possibility that readily available organic acids may be expected to have modest potentiating effects on colistin and aminoglycosides and may influence therapeutic outcomes.

\section{CONCLUSION}

Bacterial culture conditions are known to have a substantial impact on antibacterial susceptibility testing. Here, we characterize the effect of three key nutrients on the susceptibility of $P$. aeruginosa to membrane active components. In addition to the combination of $\mathrm{Ca}^{2+}$ and $\mathrm{Mg}^{2+}$ divalent cations, which induce a substantial change in cell wall composition, glucose is shown to make a small contribution to antimicrobial tolerance through an osmotic effect. Formate, a common component of bacteriological media but absent from cell culture media, is linked to metabolism of polyamines and, in common with other organic acids, was shown to have a sensitizing impact on the activity of colistin and the aminoglycoside tobramycin. The presence of organic acids in, for example, the mammalian lung suggests these might be a useful addition to future generations of media used for antibacterial susceptibility testing.

\section{METHODS}

Media. Mueller-Hinton Broth (MHB, Oxoid CM405), low salt Luria-Bertani (LB, Sigma L3397) broth, Tryptone Soya Broth (TSB, Oxoid CM0129), and Roswell Park Memorial Institute 1640 (RPMI, Gibco 51800035) broth were prepared by solubilizing the amount of powder suggested by the manufacturer in ultrapure water. RPMI is a defined medium, and its complete formulation is available from the manufacturer. The precise composition of MHB, LB, and TSB is unknown other than (1) MHB contains dehydrated infusion from beef $(300 \mathrm{~g} / \mathrm{L})$, casein hydrolysate $(17.5 \mathrm{~g} / \mathrm{L})$, and starch $(1.5 \mathrm{~g} / \mathrm{L})$; (2) $\mathrm{LB}$ contains $\mathrm{NaCl}(0.5 \mathrm{~g} / \mathrm{L})$, tryptone $(10 \mathrm{~g} / \mathrm{L})$, and yeast extract $(5 \mathrm{~g} / \mathrm{L})$; and (3) TSB contains pancreatic digest of casein $(17 \mathrm{~g} / \mathrm{L})$, enzymatic digest of soya bean $(3 \mathrm{~g} / \mathrm{L}), \mathrm{NaCl}(5 \mathrm{~g} / \mathrm{L})$, 
$\mathrm{K}_{2} \mathrm{HPO}_{4}(2.5 \mathrm{~g} / \mathrm{L})$, and glucose $(2.5 \mathrm{~g} / \mathrm{L}) . \mathrm{MHB}, \mathrm{LB}$, and TSB were autoclaved at 121 for $15 \mathrm{~min}$. Five percent of FBS was added to RPMI media, and this was filtered through a $0.22 \mu \mathrm{m}$ membrane. Cation adjusted MHB (CAMHB) was prepared according to CLSI guidelines by supplementing MHB to a final concentration of $20-25 \mathrm{mg} / \mathrm{L} \mathrm{Ca}^{2+}$ and $10-12.5 \mathrm{mg} / \mathrm{L} \mathrm{Mg}^{2+}$. Cations were added after sterilization of the media from $\mathrm{CaCl}_{2}$ and $\mathrm{MgCl}_{2}$ sterile stock solutions, as described in the guidelines. ${ }^{1}$

Bacterial Growth Conditions. E. coli NCTC 9001 and $P$. aeruginosa PAO1 were streaked on differently composed agar plates according to the media to be tested (MHB, CAMHB, LB, Tryptic Soy Agar (TSA), and RPMI with 5\% FBS) and incubated at $37{ }^{\circ} \mathrm{C}$ overnight. A further selection of five multidrug-resistant (MDR) and susceptible clinical $P$. aeruginosa isolates were streaked on agar plates composed of either Mueller-Hinton or RPMI with 5\% FBS: water supply in neonatal ward (372261), sputum (CAS2), bronchial alveolar lavage (CAS3), wound (CAS4), and cystic fibrosis (GH12, MDR) strains. Strains are representative of both of the major $P$. aeruginosa clades described by Freschi et al. with 372261, CAS2 and CAS4 being closely related to PAO1 and CAS3 and GH12 being more similar to the PA14 type strain. ${ }^{62}$ Single colonies were used to inoculate a defined volume (depending on the technique to be used) of respective media broth and incubated overnight at $37{ }^{\circ} \mathrm{C}$ without shaking. The same conditions were used to characterize the response of $P$. aeruginosa to different media components. MHB was supplemented with seven increasing concentrations of $\mathrm{Mg}^{2+} / \mathrm{Ca}^{2+}$, glucose (see Figures S5A and S6A) and RPMI with increasing concentrations of sodium formate. These broths were used to prepare $P$. aeruginosa cultures.

Antibacterial Activity Assay. Bacteria were grown overnight in $5 \mathrm{~mL}$ of respective media without shaking at $37^{\circ} \mathrm{C}$. The antibacterial activity was assessed through a modified two-fold broth microdilution assay, as described before. ${ }^{27}$ The method broadly followed EUCAST methodology with noncation adjusted Mueller Hinton, LB, TSB, and RPMI being used in addition to cation-adjusted Mueller Hinton. The $\mathrm{MIC}_{50}$ reported for each peptide in Table 1 is the average of at least three biological replicate experiments performed as follows: briefly, a two-fold dilution of peptide or antibiotic stock solutions was performed in a 96-well polypropylene microtiter plate. Then, $50 \mu \mathrm{L}$ of a bacterial suspension (back diluted from overnight cultures to an $\mathrm{A}_{620}$ of 0.001 ) were added to $50 \mu \mathrm{L}$ of peptide solution in each well of the plate reaching a final volume of $100 \mu \mathrm{L}$. Growth and sterility controls were present for each experiment. The plate was sealed with a sticker and incubated at $37^{\circ} \mathrm{C}$ for $18 \mathrm{~h}$ without shaking. Bacterial growth was observed with a colorimetric assay. Twenty microliters of a resazurin solution $(0.2 \mathrm{~g} / \mathrm{L})$ was added to each well, and the plate was incubated for an additional $2 \mathrm{~h}$. The results were read at A570 and A600 (pink and blue color, respectively) on a microplate reader (Molecular Devices SpectraMax 340 PC Microplate Reader) and plotted as the difference in absorbance versus peptide concentrations. The plot obtained was fitted with a sigmoidal curve to obtain the concentration necessary to inhibit the growth of $50 \%$ of bacteria $\left(\mathrm{MIC}_{50}\right)$. The data in Table 3 are modal MICs generated from at least three biological replicate experiments, performed as above with the following modifications: where appropriate, RPMI (5\% FBS) was doped with 40 $\mathrm{mM}$ sodium formate, sodium acetate, sodium butyrate, sodium citrate or sodium propionate; plates were incubated, static at 37
${ }^{\circ} \mathrm{C}$, for $20 \mathrm{~h}$, and the $\mathrm{OD}_{600}$ was determined using a Clariostar plate reader (BMG Labtech); the MIC was defined as the lowest concentration where growth was $<0.1$ above the background absorbance. Modification, with the sodium salt of the various organic acids, did not significantly alter the $\mathrm{pH}$ of the media.

NMR Metabolomics. On three separate occasions, three distinct colonies were picked and overnight cultures $(n=9)$ were grown in $10 \mathrm{~mL}$ of the respective media, reaching stationary phase, and pelleted at $5000 \mathrm{rpm}$ and $4{ }^{\circ} \mathrm{C}$. Supernatant was collected and filtered through $0.22 \mu \mathrm{m}$ membrane filters to remove remaining bacterial cells. The bacterial pellet was centrifuged twice at $10000 \mathrm{rpm}$ in order to remove as much media as possible. Pellets were snap frozen in liquid nitrogen before freeze-drying using an Alpha 1-2 LD plus freeze-dryer (Martin Christ, Germany) and resuspended in 40 $\mu \mathrm{L}$ of $\mathrm{D}_{2} \mathrm{O}$. The resuspended samples were placed in Kel-F insert and then in the $4 \mathrm{~mm}$ zirconia magic angle spinning (MAS) rotor and preserved with a Kel-F cap (Bruker, Rheinstetten, Germany). Data acquisition was performed on a $600 \mathrm{MHz}$ Bruker Avance III spectrometer equipped with a $4 \mathrm{~mm}$ HR-MAS probe, keeping the temperature at $310 \mathrm{~K}$. Spinning speed was 5 $\mathrm{kHz} .{ }^{1} \mathrm{H}$ NMR spectra were collected with a Carr-Purcell Meiboom-Gill presaturation (cpmgpr1d) pulse sequence with a spectrum width of $16.0 \mathrm{ppm}$ and 9615 data points using 64 transients. Free induction decay was multiplied by an exponential function with line broadening of $0.3 \mathrm{~Hz}$.

For the analysis of the spent media, $500 \mu \mathrm{L}$ of each supernatant sample was supplemented with $10 \% \quad \mathrm{D}_{2} \mathrm{O}$ containing 3-(trimethylsilyl)propionic-2,2,3,3-d4 acid sodium salt (TSP-d4) to provide a deuterium lock signal with reference signal. Samples were then transferred to NMR tubes. Samples of fresh media for each condition were treated and analyzed following the same protocol. Samples were $\mathrm{pH}$ adjusted before running NMR experiments. ${ }^{1} \mathrm{H}$ NMR spectra were recorded on a Bruker $600 \mathrm{MHz}$ Bruker Avance III NMR spectrometer (Bruker BioSpin, Coventry, United Kingdom) equipped with a 5 $\mathrm{mm}{ }^{1} \mathrm{H},{ }^{13} \mathrm{C}$, ${ }^{15} \mathrm{~N}$ TCI Prodigy Probe and a cooled sample changer with 10 sample replicates tested per condition and all kept at $4{ }^{\circ} \mathrm{C}$. The $1 \mathrm{D}$ spectra were recorded under automation at $298 \mathrm{~K}$ using a Carr-Purcell-Meiboom-Gill presaturation (cpmgpr1) pulse sequence. Spectra were acquired with 32 transients, a spectrum width of $19.83 \mathrm{ppm}$ and 65536 data points. For both liquid state and HR-MAS, correlation spectroscopy (COSY), total correlation spectroscopy (TOCSY), and ${ }^{1} \mathrm{H}-{ }^{13} \mathrm{C}$ heteronuclear single quantum correlation (HSQC) spectra were acquired for a subset of samples to aid the assignment of metabolite resonance. All peak positions were measured relative to the methyl peak of TSP-d4 set to 0.0 ppm.

Data Analysis and Statistics. The spectra were Fourier transformed automatically using standard Bruker commands and manually phased and baseline corrected in Bruker TopSpin 4.0 (Bruker Biospin, Coventry, U.K.). Metabolites were assigned using the databanks: Chenomx NMR suite software (Chenomx Inc., Canada), Human Metabolome Database (HMDB), Biological Magnetic Resonance Data Bank (BMRB), ${ }^{63}$ E. coli Metabolome Database (ECMDB), and AOCS lipid library; and a comparison of chemical shifts to the literature, which was confirmed using 2D NMR spectra. The intensities of assigned NMR peaks were analyzed using nonparametric univariate methods. Volcano plots compared the fold change in metabolite values between two conditions. Fold change was calculated as the ratio between each media and MHB. Mann-Whitney U test 
was used to compare means, and associated p-values were False Discovery rate adjusted using the Benjamini-Hochberg method $(\alpha=0.05)$. Volcano plots were generated using custom scripts in Python with Numpy, Pandas, Matplotlib, and Seaborn packages. Box plots were generated using changes in normalized intensity for each metabolite, and significant differences between challenges and controls were determined using a one wayANOVA. Data were treated with probabilistic quotient normalization (PQN) before the analysis. ${ }^{64}$ PLS Regression was performed in Python using the PLSRegression function in the scikit-learn software package. Initial model assessment determined that only one component was necessary for best model performance. Increased number of components led to a decrease in $Q^{2}$. Monte Carlo cross-validation of models was performed by randomly splitting data into 70/30 training/test set splits. Model generation and assessment was repeated 1000 times to avoid bias by sample separation and $R^{2}$ and $Q^{2}$ values were calculated to assess model performance. The $R^{2}$ metric demonstrates how well the model describes the training data set while $Q^{2}$ is a metric of how well the model predicts the test set.

Transmission Electron Microscopy. Control and challenge samples were grown as above for NMR metabolomics studies. Pellets were then washed with $2.5 \%$ glutaraldehyde in cacodylate buffer (with supplemented glucose for the RPMI sample) and fixed in the same buffer overnight at $4{ }^{\circ} \mathrm{C}$. Samples were prepared and imaged as follows: samples were osmicated in $1 \%$ osmium tetroxide in cacodylate buffer for $1.5 \mathrm{~h}$ at $4{ }^{\circ} \mathrm{C}$ and then washed twice with distilled water. Samples were stained with $1 \%$ uranyl acetate in water for $1 \mathrm{~h}$ at room temperature and washed again in distilled water before dehydrating in a graded ethanol series. Preinfiltration with propylene oxide was carried out twice, both for $10 \mathrm{~min}$, before samples were infiltrated with a series of SPURR resin/propylene oxide mixture followed by infiltration of $100 \%$ SPURR resin for $24 \mathrm{~h}$. Samples were then embedded and polymerized for $24 \mathrm{~h}$ at $60{ }^{\circ} \mathrm{C}$. Images were acquired using a JEOL JEM-1400Plus microscope at $120 \mathrm{kV}$ with a JEOL Ruby camera (JEOL, U.S.A.).

2,7-Dichlorodihydrofluorescein Diacetate (DCFH-DA) Fluorescence. $P$. aeruginosa $\mathrm{PAO} 1$ was cultured overnight at $37{ }^{\circ} \mathrm{C}$ in either MHB or RPMI (5\% FBS) in the presence and absence of either $5 \mathrm{mM}$ or $40 \mathrm{mM}$ sodium formate. Samples were pelleted by centrifugation and diluted to an $\mathrm{OD}_{600}$ of 0.6 in phosphate buffered saline (PBS). The cell suspension was incubated in darkness for $1 \mathrm{~h}$ at $37^{\circ} \mathrm{C}$ with $5 \mu \mathrm{M}$ DCFH-DA. Upon completion of incubation, the cell suspension was washed with PBS to remove excess dye and incubated in darkness at 37 ${ }^{\circ} \mathrm{C}$ for $5 \mathrm{~min}$ to equilibrate. Fluorescence spectra $(515-545 \mathrm{~nm})$ were obtained using a Varian Cary Eclipse fluorescence spectrometer at $37^{\circ} \mathrm{C}$ with excitation at $485 \mathrm{~nm}$.

\section{ASSOCIATED CONTENT}

\section{SI Supporting Information}

The Supporting Information is available free of charge at https://pubs.acs.org/doi/10.1021/acsinfecdis.1c00002.

Two additional tables (Tables S1 and S2) and 11 additional figures (Figures S1-S11) (PDF)

\section{AUTHOR INFORMATION}

\section{Corresponding Author}

A. James Mason - Institute of Pharmaceutical Science, School of Cancer and Pharmaceutical Sciences, King's College London, London SE1 9NH, United Kingdom; ㅈorcid.org/0000-
0003-0411-602X; Phone: +44 207848 4813;

Email: james.mason@kcl.ac.uk; Fax: +44 2078484800

\section{Authors}

Giorgia Manzo - Institute of Pharmaceutical Science, School of Cancer and Pharmaceutical Sciences, King's College London, London SE1 9NH, United Kingdom

Federico Gianfanti - Institute of Pharmaceutical Science, School of Cancer and Pharmaceutical Sciences, King's College London, London SE1 9NH, United Kingdom

Charlotte K. Hind - Technology Development Group, National Infection Service, Public Health England, Salisbury SP4 OJG, United Kingdom

Leanne Allison - Centre for Ultrastructural Imaging, Guy's Campus, King's College London, London SE1 1UL, United Kingdom

Maria Clarke - Institute of Pharmaceutical Science, School of Cancer and Pharmaceutical Sciences, King's College London, London SE1 9NH, United Kingdom

Julia Hohenbichler - Institute of Pharmaceutical Science, School of Cancer and Pharmaceutical Sciences, King's College London, London SE1 9NH, United Kingdom

Ilene Limantoro - Institute of Pharmaceutical Science, School of Cancer and Pharmaceutical Sciences, King's College London, London SE1 9NH, United Kingdom

Bethany Martin - Technology Development Group, National Infection Service, Public Health England, Salisbury SP4 0JG, United Kingdom

Phoebe Do Carmo Silva - Technology Development Group, National Infection Service, Public Health England, Salisbury SP4 0JG, United Kingdom

Philip M. Ferguson - Institute of Pharmaceutical Science, School of Cancer and Pharmaceutical Sciences, King's College London, London SE1 9NH, United Kingdom

Alice C. Hodgson-Casson - Institute of Pharmaceutical Science, School of Cancer and Pharmaceutical Sciences, King's College London, London SE1 9NH, United Kingdom

Roland A. Fleck - Centre for Ultrastructural Imaging, Guy's Campus, King's College London, London SE1 1UL, United Kingdom

J. Mark Sutton - Technology Development Group, National Infection Service, Public Health England, Salisbury SP4 OJG, United Kingdom; 이이.org/0000-0002-2288-0446

David A. Phoenix - School of Applied Science, London South Bank University, London SE1 OAA, United Kingdom

Complete contact information is available at:

https://pubs.acs.org/10.1021/acsinfecdis.1c00002

\section{Author Contributions}

A.J.M. wrote the main manuscript text and prepared all figures. G.M., D.A.P., R.A.F., J.M.S., C.K.H. and A.J.M. designed experiments and/or devised experimental procedures. G.M., F.G., J.H., I.L., M.C., and P.M.F. performed and/or analyzed NMR metabolomic experiments/data. M.C. performed and analyzed DCFH-DA fluorescence experiments. G.M., F.G., J.H., I.L., C.K.H., B.M., and P.D.C.S. conducted antimicrobial susceptibility testing. G.M., A.C.H.-C., and L.A. obtained electron microscopy images with RAF. All authors approved the manuscript.

Notes

The authors declare no competing financial interest. 


\section{ACKNOWLEDGMENTS}

NMR experiments described in this paper were carried out using the facilities of the Centre for Biomolecular Spectroscopy, King's College London, and at the MRC Biomedical NMR Centre at the Francis Crick Institute. The King's instruments were acquired with a Multiuser Equipment Grant from the Wellcome Trust and an Infra-structure Grant from the British Heart Foundation. The MRC Biomedical NMR Centre is supported by the Francis Crick Institute, which receives its core funding from Cancer Research UK (FC001029), the U.K. Medical Research Council (FC001029), and the Wellcome Trust (FC001029). We thank Dr. Tom Frenkiel and Dr. Alain Oregioni for their assistance with HR-MAS NMR experiments performed at the Francis Crick Institute and Dr. Andrew Atkinson for liquid-state NMR experiments performed at KCL. P.M.F. was supported by a Health Schools Studentship funded by the EPSRC (EP/M50788X/1). A.J.M. and G.M. received funding from the MRC Proximity to Discovery: Industry Engagement Fund (MC_PC_16074) and the King's Health Partners R\&D Challenge rapid fund.

\section{REFERENCES}

(1) Clinical and Laboratory Standards Institute (2015) Methods for dilution antimicrobial susceptibility tests for bacteria that grow aerobically. Approved Standard, 10th ed.; Clinical and Laboratory Standards Institute: Wayne, PA: 2015; CLSI document M07-A10 (https://clsi.org/media/1632/m07a10 sample.pdf).

(2) Kubicek-Sutherland, J. Z., Heithoff, D. M., Ersoy, S. C., Shimp, W. R., House, J. K., Marth, J. D., Smith, J. W., and Mahan, M. J. (2015) Host-dependent induction of transient antibiotic resistance: a prelude to treatment failure. EBioMedicine 2, 1169-1178.

(3) Lin, L., Nonejuie, P., Munguia, J., Hollands, A., Olson, J., Dam, Q., Kumaraswamy, M., Rivera, H., Corriden, R., Rohde, M., Hensler, M. E., Burkart, M. D., Pogliano, J., Sakoulas, G., and Nizet, V. (2015) Azithromycin synergizes with cationic antimicrobial peptides to exert bactericidal and therapeutic activity against highly multidrug-resistant Gram-negative bacterial pathogens. EBioMedicine 2, 690-698.

(4) Ersoy, S. C., Heithoff, D. M., Barnes, V. L., Tripp, G. K., House, J. K., Marth, J. D., Smith, J. W., and Mahan, M. J. (2017) Correcting a fundamental flaw in the paradigm for antimicrobial susceptibility testing. EBioMedicine 20, 173-181.

(5) Turner, K. H., Wessel, A. K., Palmer, G. C., Murray, J. L., and Whiteley, M. (2015) Essential genome of Pseudomonas aeruginosa in cystic fibrosis sputum. Proc. Natl. Acad. Proc. Natl. Acad. Sci. U. S. A. 112, $4110-4115$.

(6) Son, M. S., Matthews, W. J., Kang, Y., Nguyen, D. T., and Hoang, T. T. (2007) In vivo evidence of Pseudomonas aeruginosa nutrient acquisition and pathogenesis in the lungs of cystic fibrosis patients. Infect. Immun. 75, 5313-5324.

(7) Cornforth, D. M., Dees, J. L., Ibberson, C. B., Huse, H. K., Mathiesen, I. H., Kirketerp-Møller, K., Wolcott, R. D., Rumbaugh, K. P., Bjarnsholt, T., and Whiteley, M. (2018) Pseudomonas aeruginosa transcriptome during human infection. Proc. Natl. Acad. Sci. U. S. A. 115, E5125-E5134.

(8) Rossi, E., Falcone, M., Molin, S., and Johansen, H. K. (2018) Highresolution in situ transcriptomics of Pseudomonas aeruginosa unveils genotype independent patho-phenotypes in cystic fibrosis lungs. Nat. Commun. 9, 3459.

(9) Ibberson, C. B., and Whiteley, M. (2019) The Staphylococcus aureus transcriptome during Cystic Fibrosis lung infection. mBio 10, No. e02774-19.

(10) Frimmersdorf, E., Horatzek, S., Pelnikevich, A., Wiehlmann, L., and Schomburg, D. (2010) How Pseudomonas aeruginosa adapts to various environments: a metabolomic approach. Environ. Microbiol. 12, 1734-1747.
(11) La Rosa, R., Johansen, H. K., and Molin, S. (2019) Adapting to the airways: metabolic requirements of Pseudomonas aeruginosa during the infection of cystic fibrosis patients. Metabolites 9, 234.

(12) Kozlowska, J., Rivett, D. W., Vermeer, L. S., Carroll, M. P., Bruce, K. D., Mason, A. J., and Rogers, G. B. (2013) A relationship between Pseudomonal growth behaviour and cystic fibrosis patient lung function identified in a metabolomic investigation. Metabolomics 9, 1262-1273.

(13) Kirchner, S., Fothergill, J. L., Wright, E. A., James, C. E., Mowat, E., and Winstanley, C. (2012) Use of artificial sputum medium to test antibiotic efficacy against Pseudomonas aeruginosa in conditions more relevant to the cystic fibrosis lung. J. Visualized Exp. 6, e3857.

(14) Harrison, F., Muruli, A., Higgins, S., and Diggle, S. P. (2014) Development of an ex vivo porcine lung model for studying growth, virulence, and signaling of Pseudomonas aeruginosa. Infect. Immun. 82, $3312-3323$.

(15) Harrison, F., and Diggle, S. P. (2016) An ex vivo lung model to study bronchioles infected with Pseudomonas aeruginosa biofilms. Microbiology 162, 1755-1760.

(16) Sweeney, E., Harrington, N. E., Harley Henriques, A. G., Hassan, M. M., Crealock-Ashurst, B., Smyth, A. R., Hurley, M. N., Tormo-Mas, M.Á., and Harrison, F. (2021) An ex vivo cystic fibrosis model recapitulates key clinical aspects of chronic Staphylococcus aureus infection. Microbiology 167, No. 000987.

(17) Johansson, J., Gudmundsson, G. H., Rottenberg, M. E., Berndt, K. D., and Agerberth, B. (1998) Conformation-dependent antibacterial activity of the naturally occurring human peptide LL-37. J. Biol. Chem. 273, 3718-3724.

(18) Scott, M. G., Davidson, D. J., Gold, M. R., Bowdish, D., and Hancock, R. E. W. (2002) The human antimicrobial peptide LL-37 is a multifunctional modulator of innate immune responses. J. Immunol. 169, 3883-3891.

(19) Dürr, U. H. N., Sudheendra, U. S., and Ramamoorthy, A. (2006) LL-37, the only human member of the cathelicidin family of antimicrobial peptides. Biochim. Biophys. Acta, Biomembr. 1758, $1408-1425$

(20) Zasloff, M. (1987) Magainins, a class of antimicrobial peptides from Xenopus skin: isolation, characterization of two active forms, and partial cDNA sequence of a precursor. Proc. Natl. Acad. Sci. U. S. A. 84, $5449-5453$

(21) Ludtke, S. J., He, K., Heller, W. T., Harroun, T. A., Yang, L., and Huang, H. W. (1996) Membrane pores induced by magainin. Biochemistry 35, 13723-13728.

(22) Gregory, S. M., Pokorny, A., and Almeida, P. F. F. (2009) Magainin 2 revisited: a test of the Quantitative Model for the all-ornone permeabilization of phospholipid vesicles. Biophys. J. 96, 116131.

(23) Manzo, G., Hind, C. K., Ferguson, P. M., Amison, R. T., Hodgson-Casson, A. C., Ciazynska, K. A., Weller, B. J., Clarke, M., Lam, C., Man, R. C. H., O'Shaughnessy, B. G., Clifford, M., Bui, T. T., Drake, A. F., Atkinson, R. A., Lam, J. K. W., Pitchford, S. C., Page, C. P., Phoenix, D. A., Lorenz, C. D., Sutton, J. M., and Mason, A. J. (2020) A pleurocidin analogue with greater conformational flexibility, enhanced antimicrobial potency and in vivo therapeutic efficacy. Communications Biology 3, 697.

(24) Saint, N., Cadiou, H., Bessin, Y., and Molle, G. (2002) Antibacterial peptide pleurocidin forms ion channels in planar lipid bilayers. Biochim. Biophys. Acta, Biomembr. 1564, 359-364.

(25) Yoshida, K., Mukai, Y., Niidome, T., Takashi, C., Tokunaga, Y., Hatakeyama, T., and Aoyagi, H. (2001) Interaction of pleurocidin and its analogs with phospholipid membrane and their antibacterial activity. J. Pept. Res. 57, 119-126.

(26) Patrzykat, A., Friedrich, C. L., Zhang, L., Mendoza, V., and Hancock, R. E. W. (2002) Sublethal concentrations of Pleurocidinderived antimicrobial peptides inhibit macromolecular synthesis in Escherichia coli. Antimicrob. Agents Chemother. 46, 605-614.

(27) Kozlowska, J., Vermeer, L. S., Rogers, G. B., Rehnnuma, N., Amos, S-B.T.A., Koller, G., McArthur, M., Bruce, K. D., and Mason, A. J. (2014) Combined systems approaches reveal highly plastic responses 
to antimicrobial peptide challenge in Escherichia coli. PLoS Pathog. 10, No. e1004104.

(28) Bialvaei, A. Z., and Kafil, H. S. (2015) Colistin, mechanisms and prevalence of resistance. Curr. Med. Res. Opin. 31, 707-721.

(29) Berglund, N. A., Piggot, T. J., Jefferies, D., Sessions, R. B., Bond, P. J., and Khalid, S. (2015) Interaction of the antimicrobial peptide polymyxin B1 with both membranes of $E$. coli: a molecular dynamics study. PLoS Comput. Biol. 11, No. e1004180.

(30) Deris, Z. Z., Swarbrick, J. D., Roberts, K. D., Azad, M. A. K., Akter, J., Horne, A. S., Nation, R. L., Rogers, K. L., Thompson, P. E., Velkov, T., and Li, J. (2014) Probing the penetration of antimicrobial polymyxin lipopeptides into Gram-negative bacteria. Bioconjugate Chem. 25, 750-760.

(31) Sabnis, A., Hagart, K. L. H., Klöckner, A., Becce, M., Evans, L. E., Furniss, R. C. D., Mavridou, D. A. I., Murphy, R., Stevens, M. M., Davies, J. C., Larrouy-Maumus, G. J., Clarke, T. B., and Edwards, A. M. (2021) Colistin kills bacteria by targeting lipopolysaccharide in the cytoplasmic membrane. eLife 10, No. e65836.

(32) Wiegand, I., Hilpert, K., and Hancock, R. E. (2008) Agar and broth dilution methods to determine the minimal inhibitory concentration (MIC) of antimicrobial substances. Nat. Protoc. 3, $163-175$.

(33) Bearson, S., Bearson, B., and Foster, J. W. (1997) Acid stress responses in enterobacteria. FEMS Microbiol. Lett. 147, 173-180.

(34) Kanjee, U., and Houry, W. A. (2013) Mechanisms of acid resistance in Escherichia coli. Annu. Rev. Microbiol. 67, 65-81.

(35) Lu, P., Ma, D., Chen, Y., Guo, Y., Chen, G.-Q., Deng, H., and Shi, Y. (2013) L-glutamine provides acid resistance for Escherichia coli through enzymatic release of ammonia. Cell Res. 23, 635-644.

(36) De Biase, D., and Lund, P. A. (2015) Chapter Two - The Escherichia coli acid stress response and its significance for pathogenesis. Adv. Appl. Microbiol. 92, 49-88.

(37) Needham, B. D., and Trent, S. M. (2013) Fortifying the barrier: the impact of lipid A remodelling on bacterial pathogenesis. Nat. Rev. Microbiol. 11, 467-481.

(38) Moon, K., and Gottesman, S. A. (2009) PhoQ/P-regulated small RNA regulates sensitivity of Escherichia coli to antimicrobial peptides. Mol. Microbiol. 74, 1314-1330.

(39) Johnson, M. D., Burton, N. A., Gutiérrez, B., Painter, K., and Lund, P. A. (2011) RcsB Is required for inducible acid resistance in Escherichia coli and acts at gadE-dependent and -independent promoters. J. Bacteriol. 193, 3653-3656.

(40) Abou Alaiwa, M. H., Reznikov, L. R., Gansemer, N. D., Sheets, K. A., Horswill, A. R., Stoltz, D. A., Zabner, J., and Welsh, M. J. (2014) pH modulates the activity and synergism of the airway surface liquid antimicrobials $\beta$-defensin-3 and LL-37. Proc. Natl. Acad. Sci. U. S. A. $111,18703-18708$

(41) Wakabayashi, N., Yano, Y., Kawano, K., and Matsuzaki, K. (2017) A pH-dependent charge reversal peptide for cancer targeting. Eur. Biophys. J. 46, 121-127.

(42) Rojo, F. (2010) Carbon catabolite repression in Pseudomonas: optimizing metabolic versatility and interactions with the environment. FEMS Microbiol. Rev. 34, 658-684.

(43) Eschbach, M., Schreiber, K., Trunk, K., Buer, J., Jahn, D., and Schobert, M. (2004) Long-term anaerobic survival of the opportunistic pathogen Pseudomonas aeruginosa via pyruvate fermentation. J. Bacteriol. 186, 4596-4604.

(44) Galimand, M, Gamper, M, Zimmermann, A, and Haas, D (1991) Positive FNR-like control of anaerobic arginine degradation and nitrate respiration in Pseudomonas aeruginosa. J. Bacteriol. 173, 1598-1606.

(45) Mercenier, A., Simon, J.-P., VanderWauven, C., Haas, D., and Stalon, V. (1980) Regulation of enzyme synthesis in the arginine deiminase pathway of Pseudomonas aeruginosa. J. Bacteriol. 144, 159163.

(46) Csonka, L. N., and Hanson, A. D. (1991) Prokaryotic osmoregulation: genetics and physiology. Annu. Rev. Microbiol. 45, 569-606.

(47) Dasu, V. V., Nakada, Y., Ohnishi-Kameyama, M., Kimura, K., and Itoh, Y. (2006) Characterization and a role of Pseudomonas aeruginosa spermidine dehydrogenase in polyamine catabolism. Microbiology 152, $2265-2272$.

(48) Fine, J. (1965) Glucose content of normal urine. Br. Med. J. 1, $1209-1214$

(49) Gerich, J. E. (2003) Clinical significance, pathogenesis, and management of postprandial hyperglycemia. Arch. Intern. Med. 163, $1306-1316$.

(50) Rodríguez-Rojas, A., Baeder, D. Y., Johnston, P., Regoes, R., and Rolff, J. (2021) Bacteria primed by antimicrobial peptides develop tolerance and persist. PLoS Pathog. 17, No. e1009443.

(51) Palmer, K. L., Aye, L. M., and Whiteley, M. (2007) Nutritional cues control Pseudomonas aeruginosa multicellular behavior in cystic fibrosis sputum. J. Bacteriol. 189, 8079-8087.

(52) Ahn, S., Jung, J., Jang, I.-A., Madsen, E. L., and Park, W. (2016) Role of glyoxylate shunt in oxidative stress response. J. Biol. Chem. 291, $11928-11938$.

(53) Kwon, H. D., and Lu, C.-D. (2006) Polyamines Induce Resistance to Cationic Peptide, Aminoglycoside, and Quinolone Antibiotics in Pseudomonas aeruginosa PAO1. Antimicrob. Agents Chemother. 50, 1615-1622.

(54) Johnson, L., Mulcahy, H., Kanevets, U., Shi, Y., and Lewenza, S. (2012) Surface-localized spermidine protects the Pseudomonas aeruginosa outer membrane from antibiotic treatment and oxidative stress. J. Bacteriol. 194, 813-826.

(55) Bolard, A., Schniederjans, M., Haüssler, S., Triponney, P., Valot, B., Plésiat, P., and Jeannot, K. (2019) Production of norspermidine contributes to aminoglycoside resistance in pmrAB mutants of Pseudomonas aeruginosa. Antimicrob. Agents Chemother. 63, e01044-19.

(56) Han, M.-L., Zhu, Y., Creek, D. J., Lin, Y.-W., Anderson, D., Shen, H.-S., Tsuji, B., Gutu, A. D., Moskowitz, S. M., Velkov, T., and Li, J. (2018) Alterations of metabolic and lipid profiles in polymyxinResistant Pseudomonas aeruginosa. Antimicrob. Agents Chemother. 62, e02656-17.

(57) Zhitnitsky, D., Rose, J., and Lewinson, O. (2017) The highly synergistic, broad spectrum, antibacterial activity of organic acids and transition metals. Sci. Rep. 7, 44554.

(58) Hong, J.-H., Lee, W.-C., Hsu, Y.-M., Liang, H.-J., Wan, C.-H., Chien, C.-L., and Lin, C.-Y. (2014) Characterization of the biochemical effects of naphthalene on the mouse respiratory system using NMRbased metabolomics. J. Appl. Toxicol. 34, 1379-1388.

(59) Ciaramelli, C., Fumagalli, M., Viglio, S., Bardoni, A. M., Piloni, D., Meloni, F., Iadarola, P., and Airoldi, C. (2017) ${ }^{1} \mathrm{H}$ NMR to evaluate the metabolome of bronchoalveolar lavage fluid (BALF) in bronchiolitis obliterans syndrome (BOS): toward the development of a new approach for biomarker identification. J. Proteome Res. 16, 1669-1682. (60) Lee, S. H., Wang, T. Y., Hong, J. H., Cheng, T. J., and Lin, C. Y. (2016) NMR-based metabolomics to determine acute inhalation effects of nano- and fine-sized $\mathrm{ZnO}$ particles in the rat lung. Nanotoxicology 10, 924-934.

(61) Flynn, J. M., Niccum, D., Dunitz, J. M., and Hunter, R. C. (2016) Evidence and role for bacterial mucin degradation in cystic fibrosis airway disease. PLoS Pathog. 12, No. e1005846.

(62) Freschi, L., Bertelli, C., Jeukens, J., Moore, M. P, Kukavica-Ibrulj, I., Emond-Rheault, J.-G., Hamel, J., Fothergill, J. L, Tucker, N. P, McClean, S., Klockgether, J., de Soyza, A., Brinkman, F. S L, Levesque, R. C, and Winstanley, C. (2018) Genomic characterisation of an international Pseudomonas aeruginosa reference panel indicates that the two major groups draw upon distinct mobile gene pools. FEMS Microbiology Lett. 365, fny120 DOI: 10.1093/femsle/fny120.

(63) Ulrich, E. L., Akutsu, H., Doreleijers, J. F., Harano, Y., Ioannidis, Y. E., Lin, J., Livny, M., Mading, S., Maziuk, D., Miller, Z., Nakatani, E., Schulte, C. F., Tolmie, D. E., Kent Wenger, R., Yao, H., and Markley, J. L. (2007) BioMagResBank. Nucleic Acids Res. 36, D402-D408.

(64) Dieterle, F., Ross, A., Schlotterbeck, G., and Senn, H. (2006) Probabilistic quotient normalization as robust method to account for dilution of complex biological mixtures. Application in ${ }^{1} \mathrm{H}$ NMR metabonomics. Anal. Chem. 78, 4281-4290. 